\title{
The Respiratory System
}

\author{
Jonathan E. Rodnick and James K. Gude
}

\section{Core Problems}

Bronchitis
Chronic Obstructive Pulmonary Disease
Pneumonia
Cough

\section{Core Procedures}

\section{Chest Roentgenogram Interpretation Tuberculin Skin Test Pulmonary Function Testing Thoracentesis and Chest Intubation Gram's Stain of Infected Material}

Because of the frequency of respiratory problems, family physicians must be knowledgeable in their diagnosis and treatment. In the now classic Virginia study, ${ }^{1}$ diseases of the respiratory system constituted the greatest number of problems seen compared to other diagnostic categories. In a review of 3 years of data and more than 120,000 visits to our Family Practice Center at the Community Hospital of Sonoma County (California), we found that $13.0 \%$ of the encounters were for respiratory problems (exclusive of ear/sinus disorders). According to estimates from the National Ambulatory Care Survey in 1981, respiratory problems accounted for $17.7 \%$ of all visits to general and family physicians and $12.5 \%$ of visits to all physicians. ${ }^{2}$ Table 17.1 shows the frequency rank of common respiratory problems compared to all problems seen by family physicians from four epidemiologic studies. Each number represents the rank of how frequently respiratory problems are encountered, e.g. acute bronchitis was the fifth most common problem (of all diagnoses) seen by Virginia family physicians. The braces indicate where the study combined diagnoses. Although the data come from different populations and may use different diagnostic categories, the message is clear: These problems are commonly encountered by family physicians.

\section{Role of the Family Physician}

The fact that pulmonary diseases are common and widespread has many implications for family physicians. Below are some of the important philosophical considerations the family physician should keep in mind during the work-up and management of patients with respiratory diseases.

1. A thorough occupational history is obtained, and exposures to fumes, gases, and dusts in the environment are noted. Silicosis from mining, manufacturing, or sandblasting, coal miners' pneumoconiosis, and asbestosis can be prevented. Pulmonary fibrosis can be caused from long-term exposure to irritant gases such as cadmium, chlorine, mercury, or nitrous fumes. Organic dust from spores, fungal antigens, and avian sources can cause an interstitial pneumonitis.

2. A careful family history is obtained in regard to pulmonary diseases with a genetic predisposition. A type of emphysema related to $\alpha_{1}$-antitrypsin deficiency can be inherited. Neurofibromatosis and tuberous sclerosis often have pulmonary components. Hereditary hemorrhagic telangiectasia (Osler-Rendu-Weber) and pulmonary arteriovenous fistulas are inherited as non-sex-linked dominant traits. Cystic fibrosis is an autosomal recessive genetic disease in which both parents carry the gene but have no symptoms (because of this fact the family history can be negative).

3. Respiratory problems and diseases can frequently be prevented. For example, the physician has the opportunity and the obligation to be a strong advocate for smoking cessation. Smoking one pack of cigarettes per day shortens one's life expectancy 6 to 8 years -4 minutes for each cigarette smoked-primarily because of increased cardiovascular disease but also because of lung cancer, bladder cancer, esophageal cancer, and respiratory failure. Just mentioning the importance of stopping or mildly confronting a smoker has an impact. 
Table 17.1. Frequency ranking of respiratory problems seen by family physicians: Comparison of four studies

\begin{tabular}{|c|c|c|c|c|}
\hline Problem $^{\mathrm{a}}$ & $\begin{array}{l}\text { Virginia }^{1} \\
(1973-75)\end{array}$ & $\begin{array}{c}\text { National } \\
\text { Ambulatory } \\
\text { Medical } \\
\text { Care } \\
\text { Survey }^{2} \\
(1981)\end{array}$ & $\begin{array}{c}\text { San Bernardino } \\
(1982-83)\end{array}$ & $\begin{array}{c}\text { Santa Rosa }^{4} \\
(1982-85)\end{array}$ \\
\hline URI, unspecified & 8 & t? & $\zeta_{4}$ & 2 \\
\hline URI, febrile & 10 & \}$^{2}$ & \}$^{4}$ & \}$^{2}$ \\
\hline Pharyngitis & 4 & 6 & & 14 \\
\hline Otitis media & 11 & 8 & 6 & 3 \\
\hline Bronchitis, acute & 5 & 7 & 21 & 12 \\
\hline Pneumonia, unspecified & 25 & 45 & $\int^{21}$ & 22 \\
\hline Asthma & 30 & 36 & 8 & 15 \\
\hline Emphysema & 95 & 50 & 9 & 27 \\
\hline Cough & 83 & & & 66 \\
\hline Chest pain & 108 & 71 & 27 & 61 \\
\hline Patient visits & 526,196 & 43,366 & 44,453 & 124,887 \\
\hline
\end{tabular}

${ }^{a}$ URI $=$ upper respiratory tract infection

4. Other aspects of prevention include the administering of appropriate immunizations. Pneumococcal vaccine should be given once prior to splenectomy as well as to those with chronic respiratory or debilitating conditions to help prevent pneumococcal pneumonia. Although only partially effective, influenza vaccine should be given yearly to the elderly with chronic disease or in skilled nursing facilities.

5. Treating respiratory illnesses is an unsurpassed opportunity for patient education and for influencing and modifying behavior. Although smoking cessation overshadows all other considerations, teaching asthmatics about the causes of their disease and the need for medication compliance may reduce their need for hospitalization. Appropriate training in the use of inhalers and home oxygen also can help those with chronic obstructive pulmonary disease (COPD).

6. The family and behavioral aspects of pulmonary disease deserve special consideration. Disability from respiratory insufficiency due to silicosis or COPD has major family implications, as such patients are often dependent on others for many daily activities. As with other chronic illness, depression and sexual problems may go undiagnosed or untreated. Family and social support in smoking cessation programs is crucial.

7. Rehabilitation of those with respiratory insufficiency offers hope for improved functioning through breathing exercises and training, psychological support, and realistic goal setting.

8 . The family physician is the patient's advocate in providing continuity of care, judicious use of diagnostic and treatment modalities, and facilitating communication among the patient, the family, and consultant physicians.

\section{Role of the Chest Consultant}

The necessity for the family physician to work closely with consultants-usually pulmonologists, thoracic surgeons, and radiologists-is never more apparent than with respiratory disease. The most common reasons for asking the assistance of a pulmonary consultant are the following: (1) the management of severe respiratory failure (of any cause); (2) help with diagnosis - usually of a coin lesion, mass lesion, or pleural effusion; (3) when one's present treatment is not working as expected with COPD, pneumonia, or interstitial lung disease; (4) for treatment of unusual conditions such as cystic fibrosis, atypical tuberculosis, sarcoid or fungal lung disease; (5) the performance of a specific diagnostic or treatment procedure such as bronchoscopy or pleural biopsy, computed tomography (CT), or pulmonary resection; and (6) when patient, family, or physician needs reassurance that the patient is indeed being handled appropriately. Other factors may enter the equation of whether to ask for a consultation, including hospital guidelines, ease of access to consultants, rapport between the physician and the patient, and economic issues (e.g., whether the primary physician is in a gatekeeper role).

The most important underlying concept is communication. One needs to be as specific and detailed as possible before the patient sees the consultant. First, the need and purpose of the referral/consultation are reviewed with the patient. Then one needs to communicate with the consultant if it is a consultation ("Please see the patient one or two times and help formulate a diagnostic or treatment plan") or a referral ("Please assume care for this problem"'). If it is a referral, there are questions about whether the consultant is to take over total care of the patient or there is to be shared care and joint decision making. The more clearly the roles of all partiesprimary physician, consultant, and patient-are defined, the less is the likelihood of inappropriate decisions.

The best approach, if possible, is to write the consultant a letter or note in the chart. History, physical, and previous test results are noted, and the question to be answered or issues to be addressed are clearly specified. Copies of office notes or other relevant data are also included. If there is not time for a letter (e.g., the patient is in the intensive care unit with in- 
creasing respiratory failure), a phone call between physicians may be adequate. The family physician should receive information about follow-up from the consultant in order to provide continuity and coordination of care. A consultant who does not provide this information is jeopardizing the patient's care.

\section{Core Problems}

\section{Bronchitis}

\section{History}

Acute bronchitis is an inflammation of the trachea and bronchi. It is more common in smokers and occurs more frequently during the winter months. The definition of bronchitis is a clinical one. Acute bronchitis (or tracheobronchitis) is defined as an acute cough, usually productive of purulent phlegm without evidence of pneumonia. The phlegm may be tenacious, clear, or discolored, increased in amount, and foul tasting. Hemoptysis may occur, as bronchitis is its most common cause. The temperature may be slightly elevated (usually not above $102^{\circ} \mathrm{F}$ ), and night sweats or fatigue are common. Shortness of breath may be noticeable in those with underlying diminished pulmonary capacity. Occasionally there is pleuritic chest pain, usually induced by coughing. Wheezing can also be present, particularly in children or those with a history of respiratory allergies or obstructive airway disease. When both wheezing and a productive cough are present, the syndrome may be called asthmatic bronchitis or sometimes wheezy bronchitis in children.

\section{Physicial Examination and Laboratory Studies}

The pulmonary signs are commonly restricted to harsh breath sounds, bibasilar crepitations, and variable amounts of expiratory wheezing. Auscultatory sounds in the chest can be divided into musical and nonmusical. Musical sounds are termed wheezes and nonmusical sounds crepitations. Synonyms for crepitations include rattles, rales, and rhonchi. A deep breath that reduces atelectasis or a cough that removes secretions in the large airways may clear crepitations heard in bronchitis. The chest $\mathrm{x}$-ray film is often ordered to help differentiate bronchitis from pneumonia in those with systemic symptoms and chest findings (e.g., crepitations, which are sometimes heard in those without chest x-ray abnormalities). There is no diagnostic finding characteristic of bronchitis; usually the $\mathrm{x}$-ray film is interpreted as normal. Findings consistent with bronchitis on chest $\mathrm{x}$-ray films include thickening of the walls of large bronchi, increased pulmonary markings in the lower lobes, and overinflation. With underlying lung disease, sputum culture is a consideration.

The etiologies of acute bronchitis are numerous, and it is frequently impossible (and seldom necessary) to define the specific cause. To make an educated guess, one must be aware of the patient's history and findings as well as the epidemiology. Etiologies are listed in Table 17.2. Viral is the most frequent and almost any respiratory virus can cause bronchitis. The three most common nonviral, infectious causes are Hemophilus influenzae, Streptococcus pneumoniae, and Mycoplasma pneumoniae. The role infection plays in exacerbations of chronic bronchitis is not always clear.
Table 17.2. Common causes of tracheobronchitis

External stimuli
Tobacco
Cannabis
Air pollution
Ammonia
Industrial fumes, dust
Bacteria
H. influenzae
S. pneumoniae
Branhamella catarrhalis
Bordetella pertussis
Viruses
Respiratory syncytial
Influenza A \& B
Parainfluenza
Rhinoviruses
Adenoviruses
Coronaviruses
Other
Mycoplasma pneumoniae
Chlamydia sp.

\section{Treatment}

The treatment of acute bronchitis is still empiric. Obviously, if the patient smokes, the time is opportune for counseling. Codeine-containing cough suppressants may be indicated in those with significant nighttime cough. Glyceryl guaiacolate, potassium iodide, mucolytic agents, antihistamines, and decongestants are usually not helpful. If influenza $A$ is suspected, amantadine $100 \mathrm{mg}$ b.i.d. is indicated, either as prevention or within the first 48 hours of symptoms.

The role of antibiotics in the treatment of acute bronchitis or an acute exacerbation of chronic bronchitis is controversial. Unfortunately, most studies of bronchitis treatment were not done as randomized blinded trials. Of four well-designed studies, two found no benefit from a 1-week course of doxycycline, although there was a reduction in subsequent new upper respiratory tract infections ${ }^{5,6}$; one found some improvement in cough and time off work when patients were treated with trimethoprim-sulfamethoxazole ${ }^{7}$; and one found no difference with a 1-week trial of erythromycin, although paradoxically nonsmokers were helped more than smokers. ${ }^{8}$

The antibiotics that might be reasonable choices for therapy are listed in Table 17.3 along with their antibacterial spectrum. Unfortunately, none is effective against all the common bacterial pathogens. The choice of whether to use an antibiotic and, if so, which one still rests with clinical judgment. At this time the consensus is not to treat those who are otherwise healthy and who have no significant systemic symptoms. Bronchitis is a self-limited condition with a course that probably is not dramatically changed by antibiotics. For those with preexisting conditions, e.g., diabetes, COPD, or congestive heart failure, and who have a productive cough along with other symptoms, most physicians lean toward treating for 1 to 2 weeks with one of the drugs listed in Table 17.3 with the hope of decreasing sputum production and perhaps preventing the occasional progression toward pneumonia or respiratory failure. 
Table 17.3. Oral antibiotics used to treat bronchitis

\begin{tabular}{lccc} 
& \multicolumn{3}{c}{ Sensitivities } \\
\cline { 2 - 4 } \multicolumn{1}{c}{ Drug } & H. influenzae & Streptococcus sp. & Mycoplasma sp. \\
\hline Tetracycline & \pm & \pm & + \\
Penicillin & - & + & - \\
Ampicillin* & \pm & + & - \\
Erythromycin & - & + & + \\
Sulfa/trimethoprim & + & + & - \\
Cefaclor & + & + & - \\
\hline
\end{tabular}

* Resistant strains of $H$. influenzae are susceptible to amoxicillin/clavulanate potassium

In terms of cost-effectiveness, the prevention of hospitalization justifies the use of many antibiotics in outpatients. Additional therapies to be considered for those who are not responding to antibiotics include steroids and inhaled bronchodilators. Occasionally, hospitalization for parenteral therapy, fluid replacement, and supplemental oxygen is necessary. The issue of whether prophylactic antibiotics decreases exacerbations in those with chronic bronchitis or bronchiectasis is unresolved, but this treatment should be considered in certain elderly or chronically ill patients. ${ }^{9,10}$

\section{Chronic Obstructive Pulmonary Diseases}

The term "emphysema" was coined by R. T. H. Laennec in 1819 in A Treatise on the Diseases of the Chest. He also described "pulmonary catarrh" or chronic bronchitis. Emphysema, chronic bronchitis, and poorly reversible asthma comprise the clinical disorder chronic obstructive pulmonary diseases (COPD).

Emphysema, a word derived from the Greek language and meaning "an inflation," is an anatomic description of alveolar wall destruction and fusion with a loss of elastic tissue. Chronic bronchitis is a clinical disorder with excessive mucus and a chronic cough on most days for a minimum of 3 months each year for not less than 2 successive years. Asthma is a disorder characterized by reversible airways obstruction with bronchial hyperreactivity. Emphysema and chronic bronchitis affect about $3 \%$ of the population, and asthma involves about $6 \%$.

\section{Clinical Picture}

The emphysematous patient, the so-called pink puffer, is asthenic with a quiet chest and marked dyspnea on exertion. There is no coughing or sputum production. The chronic bronchitis patient, the so-called blue bloater, presents with dusky lips and fingernails, a noisy chest full of crepitations and wheezing, and the complaints of chronic sputum production, coughing, and dyspnea on exertion. Most COPD patients are blends of these two extremes and also have an asthmatic-type hyperreactivity of their airways. The approach is to define the relative contribution of each of these components and then design an appropriate intervention.

\section{Laboratory Studies}

The most important prognosticator of COPD mortality is the forced expired volume in 1 second $\left(\mathrm{FEV}_{1}\right)$ after a nebulized bronchodilator. ${ }^{11}$ This value may be seen either as an absolute value or as a percent of the forced vital capacity (see Core Procedures, Pulmonary Function Testing, below). For example, if the $\mathrm{FEV}_{1}$ is $<1$ liter, there is severe obstructive disease. Determining the percent reversibility after the administration of a beta- 2 bronchodilator is also necessary. The $\mathrm{FEV}_{1}$ is the best test to detect improvement, progression, or stability of COPD. Lung volumes, if measured, show an increase in the total lung capacity with a decrease in the forced vital capacity and an increase in the residual volume with air trapping. The carbon monoxide diffusion capacity, a reflection of the pulmonary vascular bed, can distinguish emphysema, in which the carbon monoxide diffusion capacity is reduced, from asthma and chronic bronchitis, where generally the carbon monoxide diffusion capacity is near normal or increased.

Arterial blood gas assays measure $\mathrm{Po}_{2}, \mathrm{pH}, \mathrm{PCO}_{2}$, and $\mathrm{HCO}_{3}$. COPD commonly reduces the $\mathrm{Po}_{2}$ by ventilation-perfusion mismatching and lowers the $\mathrm{pH}$ by carbon dioxide retention. $\mathrm{A} \mathrm{PO}_{2}$ of $<55 \mathrm{~mm} \mathrm{Hg}$ merits oxygen therapy, and a $\mathrm{pH}$ of $<7.25$ due to respiratory acidosis may require mechanical ventilation.

The chest $\mathrm{x}$-ray film is normal with most early COPD. With advanced COPD, the chest $\mathrm{X}$-ray studies show flattened diaphragms, increased lung sizes, hyperlucent lungs, and a small heart. The $\mathrm{x}$-ray films of chronic bronchitis often show an enlarged heart with prominent pulmonary arteries. An electrocardiogram can show right atrial enlargement, right ventricular enlargement, and a rightward axis. Polycythemia may indicate chronic hypoxemia. Other pulmonary function tests, e.g., elastic recoil and exercise studies, are the province of respiratory physiologists.

\section{Treatment}

Stopping smoking is the most important intervention. At any stage of COPD, quitting smoking retards the rate of progression of airways obstruction. ${ }^{12}$ As many as $20 \%$ of smokers quit smoking on the advice of their physicians. ${ }^{13}$ Table 17.4 lists some suggestions for both physicians and patients to help them stop smoking. One must be unequivocal with advice; simply cutting down may lead to deeper inhaling, with no reduction of inhaled tars and nicotine.

Nicotine chewing gum helps patients who have quit smoking not to restart. One slowly chews a stick of gum, instead of smoking a cigarette, up to 15 times daily as needed. The use of nicotine gum is tapered after about 3 months, and it should not be used longer than a year. The use of nicotine gum is contra- 
Table 17.4. Smoking cessation suggestions

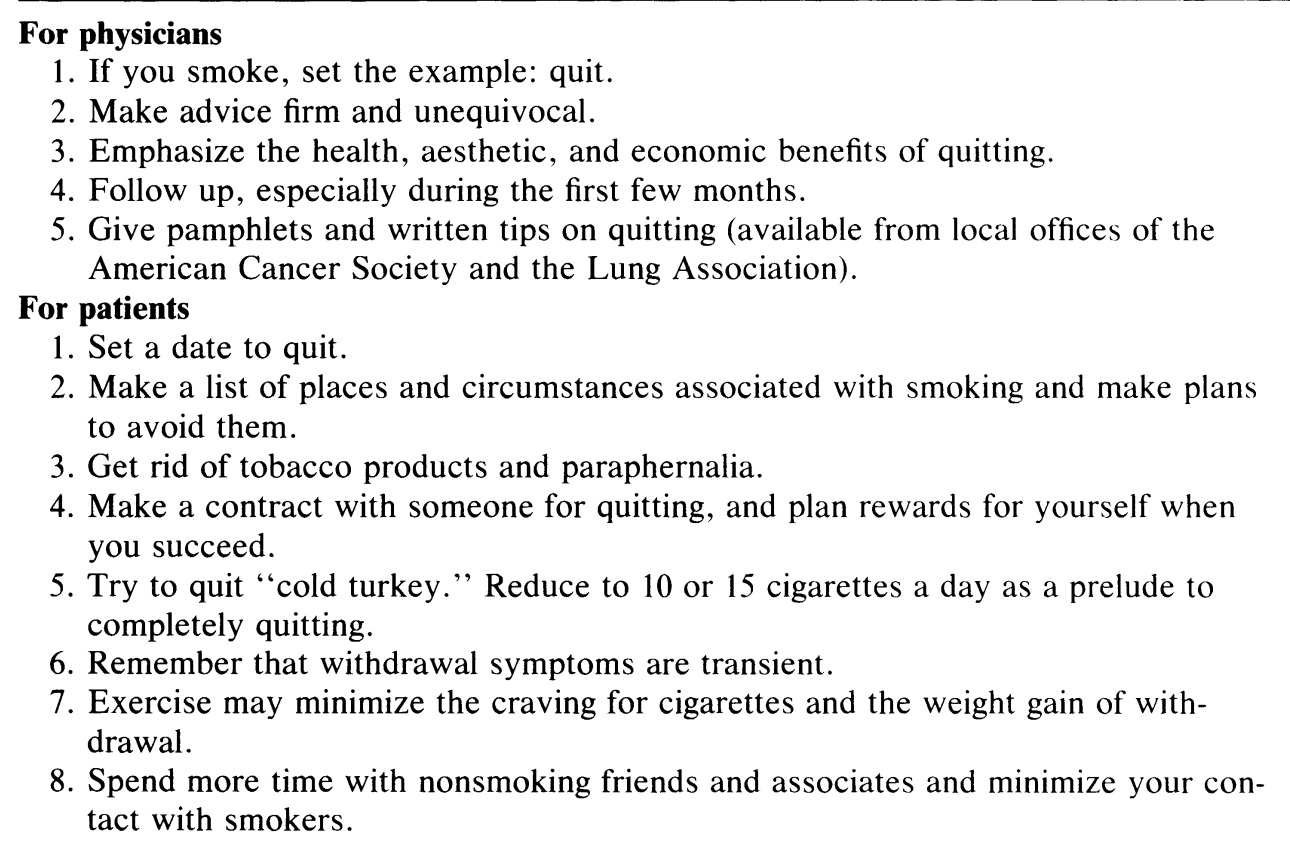

indicated during pregnancy and in patients with peptic ulcer disease or severe ischemic cardiovascular diseases. ${ }^{14}$

It has been shown that there is a high incidence of bronchial hyperreactivity with emphysema and chronic bronchitis, and that bronchodilator therapies often help. ${ }^{15}$ In general, asthma is most reversible, chronic bronchitis has intermediate reversibility, and emphysema has the least reversibility. However, one should not be dissuaded by pulmonary function tests that show little reversibility, as often a therapeutic trial of a bronchodilator or steroids brings out reversibility.

The initial drug of choice is a beta-adrenergic agonist. These effective medications are best administered to adults by inhalation, as there are fewer side effects with this route, particularly tremors. There is some added effect when tablets are given as well. Inhaled beta- 2 agonists are generally prescribed by a metered-dose inhaler. Pump nebulizers are prescribed less often. There generally is no reason to prescribe intermittent positive-pressure breathing machines to deliver these medicines. The patient is instructed to hold the inhaler several inches away from the mouth, exhale slowly, and then activate the inhaler while slowly inspiring. Five seconds of breath holding follows. The generally prescribed two puffs are better taken several minutes apart than on successive breaths. Most times two inhalations from a metered dose are sufficient, but at other times it may take more. There is no one beta- 2 agent of choice. Common agents include isoetharine, metaproterenol, terbutaline, albuterol, and bitolterol. Epinephrine and isoproterenol are generally avoided because of their cardiovascular side effects. ${ }^{16}$ Aminophylline can also be helpful in COPD patients, not only by decreasing airways obstruction but also by improving diaphragmatic contractility. ${ }^{17}$

Steroids are potent agents in COPD even when spirometry testing shows no reversibility. Generally, when other measures have failed, 30 to $60 \mathrm{mg}$ of prednisone taken for approximately 6 days reveals if there is reversibility, but objective testing with spirometry is needed to be certain of improvement, as steroids have a potent euphoric effect. ${ }^{18}$ If a brief trial of oral steroids objectively helps, there are several alternatives. First, nebulized steroids are preferred given their lack of side effects. Two to eight inhalations are used twice daily after two puffs of a beta- 2 agonist. Occasionally higher doses are needed: up to 40 puffs daily. Spacer devices improve the efficacy and decrease the oral side effects of inhaler steroids and beta-2 agonists. If inhaled medications are not effective, intermittent (every other day) steroids or daily steroids at the lowest helpful dose are the last resort.

Although commonly prescribed with bronchitic flares in COPD patients, antibiotics generally do not appear to help..$^{19}$ Anticholinergic medications, e.g., atropine and ipratropium bromide, are as useful for COPD patients as bronchodilators. ${ }^{20}$ They are synergistic with the beta- 2 agents and may be added when beta- 2 therapy alone does not suffice. Cromolyn sodium appears effective mainly in asthmatics, and it is usually not helpful with emphysema or chronic bronchitis. There are no reliable respiratory stimulants for the treatment of COPD.

Supplemental oxygen is indicated if the arterial $\mathrm{Po}_{2}$ on room air is $\leq 55 \mathrm{~mm} \mathrm{Hg}$ or if it is $\leq 59$ and associated with edema, a hematocrit $>55 \%$, or p pulmonale on ECG. It appears that oxygen for 24 hours daily at low flow rates of 1 to $2 \mathrm{l} / \mathrm{min}$ has better survival results than overnight oxygen, and both of these regimens are better than room air. ${ }^{21}$ For patients with the above criteria who do not use oxygen continuously, the use of nocturnal oxygen for 15 hours at least helps, if it does not equal, the continuous oxygen therapy results. Oxygen concentrators at low flow rates are the most economical means of delivering home oxygen.

Pulmonary rehabilitation has much to offer. ${ }^{22}$ A team approach is recommended. Exercising, not only of a general nature but specifically of the respiratory muscles, helps; and 
use of an inspiratory muscle trainer (e.g., a P flex whistle) for 15 minutes twice a day against respiratory resistance can help strengthen failing diaphragms. ${ }^{23}$

\section{Pneumonia}

Giovanni Battista Morgagni (1682-1771) published a classic treatise in 1761, The Seats and Causes of Diseases Investigated By Anatomy, in which clinical and pathologic findings of pneumonia were thoroughly presented. Pneumonia is defined as an inflammation of the lung with consolidation. During the early twentieth century pneumonias were the most common cause of death, whereas now they rank approximately seventh. Pneumonias are more common in the very young and the very old. Risk factors include age (infants and elderly), concurrent diabetes mellitus, alcoholism, COPD, congestive heart failure, and hematologic or immunologic abnormalities. Persons who have had splenectomy are especially prone to pneumococcal infections.

There are no recent epidemiologic studies on the causes of outpatient or community-acquired pneumonia treated in the physician's office. Estimates are that pneumonia develops in more than three million Americans annually. About $80 \%$ of these episodes are due to viruses, Mycoplasma, or unknown etiologies, and the remaining $20 \%$ are due to bacteria. ${ }^{24}$

If one looks only at patients in a hospital, the causes of diagnosed pneumonias can be broken down as follows ${ }^{25}$ :

Percent of infectious

Pathologic agent pneumonias

\begin{tabular}{lr}
\hline Pneumonococcus & 21.0 \\
Staphylococcus & 3.4 \\
Hemophilus influenzae & 3.2 \\
Klebsiella & 3.0 \\
Pseudomonas & 2.7 \\
Legionella & 2.7 \\
Other bacteria & 12.6 \\
& \\
Mycobacterial & 4.0 \\
Other fungal & 13.2 \\
& \\
Influenza & 3.4 \\
Cytomegalovirus & 1.5 \\
Nonspecific viral & 28.0 \\
& \\
Mycoplasma & 3.4 \\
Pneumocystis & 2.1 \\
Chlamydia & 1.8 \\
Parasitic & 0.5 \\
\hline
\end{tabular}

Reprinted with permission from ref. 25.

This adds to more than $100 \%$ as a few patient had more than one etiology. The spectrum seen in family practice is likely to be different, but epidemiologic studies are lacking.

\section{Laboratory Studies}

With symptoms of fever, cough, breathlessness, and chest pain, pneumonia must be considered. A complete blood count and chest $\mathrm{x}$-ray films are indicated. With bacterial pneumonias the white blood cell count is usually elevated with a shift to the left. The chest film shows infiltrates. The clinical question is how to proceed from this point in terms of diagnosis and antibiotic selection. In any setting with a community-acquired pneumonia, a sputum Gram's stain is important to make a tentative diagnosis (see Core Procedures, Gram's Stain, below). Bacteria of the same morphology dominating more than half of the organisms seen on the slide are interpreted as pathogens, especially if they are in or associated with polymorphonuclear leukocytes. If there are no predominant bacteria on the Gram's stain, the interpretation would be one of no obvious pathogen.

Cultures are prepared. Initial antibiotic therapy can later be changed if the clinical course so indicates. Blood cultures are definitive but are generally negative. The best culture is that of a direct lung aspirate, but that procedure is seldom done because of the risks of worsening an already ill patient with the complication of a pneumothorax or pulmonary hemorrhage. Lung aspirates by transcutaneous needle appear safe if done with small needles under fluoroscopic guidance; alternatively, special bronchoscopic techniques are available for lung secretion cultures.

Transtracheal aspirates are useful. They have not been used frequently because of the possibility of bleeding or pneumothorax. The complication rate is small in terms of a pneumothorax or significant tracheal bleed. Despite their value reported in the literature, transtracheal aspirates are generally reserved for comatose patients with pneumonia and those in whom one critical culture is needed before beginning antibiotics. Sputum cultures are rarely helpful in the diagnosis of bacterial pneumonia. ${ }^{26}$

\section{Specific Pneumonias}

\section{Pneumococcal Pneumonia}

A viral respiratory illness often precedes pneumococcal pneumonia. Aspiration of upper airway pneumococci into the alveoli appears to initiate the pneumonic process. Three-fourths of the time the patient has one rigor or shaking chill. The temperature varies between $101^{\circ}$ and $106^{\circ} \mathrm{F}$. A dry cough gives way to rusty sputum. Often a pleural friction rub is accompanied by pleuritic chest pain. The consolidated areas of the lung often produce dullness with increased bronchial breath sounds and egophony.

Chest $\mathrm{x}$-ray films generally show consolidation involving one or two lobes. The more lobes involved, the higher is the mortality. Infiltrates take up to 6 weeks to resolve. Small pleural effusions are common. Empyema is infrequent. Usually tapping these effusions is not necessary. Leukopenia can occur in very ill patients. Pneumococcal pneumonia can be accompanied by hyperbilirubinemia, in part due to hemolysis. Hypoxemia is common. Oximeters are now available that easily determine the need for oxygen and, if needed, the oxygen flow rate required to keep the oxygen-hemoglobin saturation $\geq 90 \%$. Hypercapnia is unusual unless the patient has severe underlying COPD.

In general, the mortality rate for single lobe disease is quite low, less than $5 \%$, whereas the mortality rate for involve- 
ment of multiple lobes can approach 50\%. The complications of pneumococcal bacteremia include pericarditis, meningitis, arthritis, and other distal abscesses. Treating early pneumococcal pneumonia with penicillin produces dramatic results. Significant clinical resolution occurs within 24 hours. If therapy is delayed, the disease may follow its natural crisis and lysis over 7 to 10 days.

A pneumococcal polyvalent vaccine is available that contains 23 capsular pneumococcal antigens that are responsible for approximately $85 \%$ of pneumococcal bacteremias. The vaccine is safe, with minimal side effects; it is recommended for patients with asplenia, sickle cell anemia, alcoholism, COPD, congestive heart failure, cirrhosis, diabetes méllitus, nephrotic syndrome, and renal failure. ${ }^{27}$

\section{Staphylococcal Pneumonia}

Patients with staphylococcal pneumonia should be hospitalized. Patients are generally febrile, and pleuritic chest pain is common, associated with a rub and effusion. Shaking chills occur repetitively, and there is often gross hemoptysis. These patients are significantly hypoxemic. The chest $x$-ray films reveal multiple consolidations, giving the impression of a diffuse bronchopneumonia. Despite early and appropriate antibiotic therapy, these necrotizing pneumonias tend to be protracted with complications including lung abscesses, empyemas, pneumothoraces, and bronchopleural fistulas. The clinician must always be alert for distant abscesses secondary to bactermia; prolonged treatment with antibiotics and drainage is required if such complications occur. ${ }^{28}$

\section{Gram-Negative Pneumonias}

Common causes of gram-negative pneumonia include Klebsiella pneumoniae, Hemophilus influenzae, Enterobacter species, Escherichia coli, Pseudomonas aeruginosa, and Proteus species. These pneumonias result from abnormal colonization of the oropharynx complicated by aspiration into the alveoli, producing a pneumonia. The initial sputum Gram's stain is helpful. The best cultures are of the blood, lung, pleural effusion, or transtracheal aspirate. These gram-negative pneumonias are clinically similar, and the patients' presentations of symptoms and signs are not different enough to pinpoint etiologies. The chest film is not helpful in terms of which organism is at hand ${ }^{29}$ These pneumonias are most often seen in individuals with other underlying abnormalities, and hospital admission is usually indicated.

\section{Treatment of Bacterial Pneumonias}

The initial treatment is based on the Gram's stain; if it shows a predominance of pneumococci, penicillin is the drug of choice. With a penicillin allergy, erythromycin is the alternative. If the Gram's stain shows no or few organisms, erythromycin or alternatively tetracycline is the antibiotic of choice to cover Mycoplasma pneumoniae or Legionella. If the Gram's stain shows small gram-negative rods resembling $\mathrm{He}$ mophilus influenzae, ampicillin, amoxicillin with clavulanate potassium, or cefaclor is indicated depending on the sensitivity tests. If the Gram's stain shows large gram-positive grapelike cocci, staphylococcal pneumonia may be present; and intravenous nafcillin, oxacillin, or vanomycin is indicated in a hospital setting given the virulence of staphylococcal pneumonias. If large gram-negative bacilli such as Klebsiella are seen and the patient requires hospitalization, possible antibiotic regimens include an aminoglycoside and a third generation cephalosporin, or ticarcillin with clavulanic acid. If outpatient treatment seems reasonable, amoxicillin with clavulanic acid is the treatment of choice. If the Gram's stain shows a mixed picture, treat as for $H$. influenzae (Table 17.5).

\section{Other Pneumonias}

Anaerobic bacteria deserve special mention, as they are part of the normal flora of the upper respiratory tract but, if aspirated, may produce pneumonia with or without abscesses and empyemas. An anaerobic lung abscess generally presents with putrid-smelling phlegm, and commonly the patient is alcoholic or has poor dental hygiene. Putrid abscesses generally respond to penicillin; but given an increasing incidence of renicillin resistance in Bacteriodes and other anaerobes, alternative therapies include clindamycin, cefoxitin, metronidazole, and chloramphenicol. Extended treatment may be necessary. Certain other pneumonias tend to cavitate and produce lung abscesses, and the pneumonias most prone to cavitate include those caused by staphylococci and Klebsiella. With lung abscesses, mechanical obstructions due to foreign bodies or cancers must be ruled out. Bronchoscopy is generally the diagnostic and at times the therapeutic choice if mechanical obstruction is a consideration.

Empyema is pus in the pleural cavity. The most common cause of an empyema is the extension of pneumonia into the pleural space. The most common offending organisms are the staphylococci, pneumococci, and anaerobic bacteria. Tuberculosis is always a consideration with exudative pleural effusions.

The aspiration of pleural pus is diagnostic, and if the pus has a putrid smell an anaerobic infection is present. Drainage is

Table 17.5. Outpatient pneumonia treatment

\begin{tabular}{lll}
\hline $\begin{array}{c}\text { Suspected } \\
\text { pneumonia }\end{array}$ & \multicolumn{1}{c}{ First choice } & Alternative \\
\hline $\begin{array}{l}\text { Pneumococcal } \\
\text { Mycoplasmal }\end{array}$ & $\begin{array}{l}\text { Penicillin } \\
\text { Erythromycin }\end{array}$ & $\begin{array}{l}\text { Erythromycin } \\
\text { Tetracycline }\end{array}$ \\
& $\begin{array}{c}\text { Ampicillin or amox- } \\
\text { icillin if }<20 \% \text { com- } \\
\text { munity resistance; } \\
\text { cefaclor or amox- }\end{array}$ & $\begin{array}{c}\text { Trimethoprim- } \\
\text { sulfa }\end{array}$ \\
& $\begin{array}{l}\text { icillin/clavulanate } \\
\text { potassium if } \geq 20 \%\end{array}$ & \\
& community resis- & \\
& tance to ampicillin & \\
Gram-negative & Ampicillin with clavu- & First or second \\
& lanate potassium & generation \\
& cephalosporin \\
Legionella & Erythromycin & None \\
Aspiration & Penicillin & Clindamycin or \\
& & metronidazole \\
\hline
\end{tabular}


the most important aspect of treating an empyema. Often the empyema sites are loculated, and multiple chest tubes may be needed. If there are too many loculated areas to drain by chest tubes, an open thoracotomy with the breaking up of the various adhesions and open drainage comprises definitive care. Antibiotics are less important than drainage in treating empyemas.

Mycoplasma pneumoniae is particularly common in children and young adults. The clinical markers are a dry cough and headache with no rhinorrhea. Earache occurs frequently. Extrapulmonary manifestations are surprisingly common and include myalgias, rashes, myocarditis, pericarditis, meningitis, nephritis, and hemolytic anemia. Gastrointestinal upsets are common and take weeks to resolve. Cold agglutinins are present in 40 to $80 \%$ of these patients with titers $\geq 1: 64$, but the definitive laboratory diagnosis requires a fourfold rise of specific complement fixation titers. Chest x-ray films show patchy bronchopneumonia in the lower lobes. Erythromycin and tetracycline remain the antibiotics of choice. Epidemics of this atypical pneumonia occur in young people in institutional settings. The $M$. pneumoniae organism can be isolated from sputum, pharynx, or blood, but it requires a special laboratory. ${ }^{30}$

Legionnaire's disease is often not thought of in the differential diagnosis of pneumonia. Its true incidence is unknown. Legionella pneumophilia, a gram-negative bacillus, causes legionnaire's disease. A fourfold rise in the indirect fluorescent antibody titer to $>1: 128$ is diagnostic. The clinical and $\mathrm{x}$-ray findings are variable. Therapy is erythromycin. ${ }^{31}$

Chlamydia trachomatis presents as an interstitial pneumonia during infancy with gradual onset and a protracted course. It also is a recognized cause of pneumonia and bronchitis in adults. $^{32}$ The clinical hallmarks in infants are conjunctivitis, rhinitis, and a staccato cough. Fever is usually absent. $C$. trachomatis can be cultured from nasopharyngeal aspirates and the birth canal. Treatment for children is sulfisoxazole or erythromycin. ${ }^{33}$ Another strain of Chlamydia is a recognized cause of pneumonia and bronchitis in adults and is best treated with tetracycline. ${ }^{32}$

Pneumocystis carinii deserves mention as it is frequently diagnosed in patients with acquired immune deficiency disease (AIDS). In the clinical setting of AIDS, an induced sputum or bronchoscopy with bronchial lavage generally establishes the diagnosis. At present the antibiotics of choice are trimethoprim-sulfamethoxazole or pentamidine. Pneumocystis pneumonia can be temporarily treated in the setting of AIDS, but it usually recurs. Cytomegalovirus also has been found frequently in AIDS pneumonias, but there is no specific therapy.

The most common viral pneumonia is influenza $\mathrm{A}$ or $\mathrm{B}$. Other common causes of viral pneumonias include respiratory syncytial virus and parainfluenza virus in children, and adenovirus in adults. After the typical "flu" symptoms of fever and chills, myalgias, headache, and anorexia, and accelerating course of cough, breathlessness, and cyanosis in conjunction with an $\mathrm{x}$-ray film showing a diffuse interstitial process suggests an influenzal pneumonia. A sputum Gram's stain is unrevealing. Influenza titers rise rapidly. Secondary bacterial penumonias may occur including Streptococcus pneumoniae and Staphylococcus aureus. Amantadine is indi- cated in uncomplicated acute influenza A pneumonia. Adenine arabinoside (ara-A) can help in the course of herpes varicella-zoster pneumonias. For most viral pneumonias, the treatment is supportive.

Table 17.5 summarizes the outpatient treatment of pneumonias.

\section{Cough}

\section{Diagnosis}

As with many clinical evaluations, the history and physical examination generally establish the cause of coughing without additional tests. Eighty percent of cases are clear after the history and physical are done; only $20 \%$ require diagnostic testing. ${ }^{34}$

The time of day the cough is most bothersome may be helpful. Nighttime cough with choking suggests aspiration. Cough that awakens one at night with breathlessness suggests pulmonary edema. A postnasal drip and bronchitic-type coughing usually occur in the morning. Exercise-induced cough suggests allergic asthma or interstitial lung disease. Certain allergy exposures produce not only an asthmatic reaction with wheezing and breathlessness but also coughing as the primary symptom. The cough that follows a viral illness (postinfectious) can last 2 to 3 months. A lack of wheezing, especially in children, does not rule out asthma as a cause of coughing. ${ }^{35}$

Medications need to be reviewed, as certain ones may produce coughing. Beta-blockers can produce an asthmatic reaction. Captopril is another potential offender because angiotensin-converting enzyme inhibition produces kinins, which may stimulate coughing. Drugs that may produce an interstitial lung reaction that can lead to coughing include nitrofurantoin, hydralazine, and procainamide.

It is important to listen to the patient coughing to help determine the type of cough. A dry, hacking cough suggests tracheobronchitis. A barking cough suggests an infectious cause such as croup. A wet cough suggests bronchitis. A highpitched, wheezy, tight cough suggests asthma. A cough with halitosis suggests bronchiectasis or lung abscess. On physical examination, a cobblestone mucosa and mucoid or mucopurulent secretions of the nasal or oral pharynx suggest postnasal dripping, usually due to a perennial nonallergic rhinitis. Occasionally wax or irritating hairs in the external auditory meatus can produce coughing.

The chest $\mathrm{x}$-ray film usually is normal in patients with chronic cough, but it should be obtained in doubtful cases to make sure there is no cancer, interstitial lung disease, or mass lesion producing cough. Spirometry may be helpful in the work-up of persisting cough, but it should be borne in mind that spirometry is normal in about $20 \%$ of the asthmatic patients with coughing. A challenge inhalation test with methacholine can bring out an asthmatic tendency.

\section{Therapy}

With a specific diagnosis, specific therapy is successful in more than $90 \%$ of patients. ${ }^{34}$ For many patients the most important treatment is to stop smoking. It takes 1 to 4 months off cigarettes for the cough to improve, and initially some patients' coughs worsen. With postnasal dripping, an effective treatment is to wash the sinuses twice a day with saline lavage 
to help remove irritating dusts or antigens. One should take a pint of warm water, add a teaspoon of salt, and then sniff this mixture from the cupped hand, after which one may use locally inhaled corticosteroids or cromolyn sodium. The use of antihistamines and decongestants is sometimes helpful in the treatment of postnasal dripping. Post infectious cough is treated with cough suppressants, and extended codeine preparations can be used twice daily. With asthma, oral steroids may dramatically decrease coughing. If so, inhaled steroids may prove helpful. If inhaled steroids are too irritating, a spacer device to allow the "raining out" of large droplets may prove helpful.

There are many ineffective therapies. There is no role for commercial cough preparations, which are combinations of antitussives, expectorants, sympathomimetics, and antihistamines. Although glycerol guaiacolate is often prescribed, it is no more effective than placebo in patients with the cough of chronic bronchitis, and in fact it can interfere with platelet function and produce increases in the activated plasma clotting time. Iodides have no role in the care of coughing because of their multiple side effects and unpleasant metallic taste. Water also has no role in the treatment of coughs unless the patient is greatly dehydrated, and the use of water by ultrasonic nebulizer delivers little water beneath the vocal cords. ${ }^{36}$

\section{Other Problems}

\section{Lung Cancer}

Giovanni Battista Morgagni first clearly described postmortem lung cancer in 1761. Sir William Osler in his 1892 Principles and Practice of Medicine emphasized that primary lung cancer was exceedingly rare. By contrast, lung cancer is now the leading cause of cancer death in men, and it has replaced breast cancer as the number one cause of cancer death in women. Lung cancer has reached an epidemic status in the Western world. About $90 \%$ of lung cancers belong to the category "bronchogenic carcinoma." The four major cell types are adenocarcinoma, squamous cell carcinoma, small cell carcinoma, and large cell carcinoma. Despite surgical, chemotherapeutic, and radiation treatments for lung cancer, the 5year survival rate remains only 5 to $10 \%$. Unfortunately, most patients who present with lung cancer have incurable disease.

Squamous cell carcinomas comprise about $30 \%$ of bronchogenic carcinomas, and they generally begin in the hilar areas, although about one-third start peripherally. They tend to produce central obstruction of bronchi with resultant wedgeshaped peripheral shadows. Squamous cell carcinomas are the most common cause of occult lung cancers. It is estimated that it takes more than 5 years for a squamous cell carcinoma to reach a coin-size lesion $1 \mathrm{~cm}$ in diameter.

Adenocarcinomas, including bronchioloalveolar cell carcinomas, comprise about $25 \%$ of bronchogenic cancers. They usually present on the chest x-ray film as peripheral tumors, and they tend to spread hematogenously to the central nervous system. When bronchial in origin, they are almost impossible to distinguish cytologically from metatastic adenocarcinomas (e.g., colon or pancreatic).

Small cell, or oat cell, carcinomas represent about $20 \%$ of bronchogenic carcinomas, and small cell cancers have the strongest association with cigarette smoking. Small cell carcinomas have been associated with the poorest outcomes of all bronchogenic carcinomas, but as chemotherapy improves this prognosis is changing. Small cell carcinomas are often associated with paraneoplastic syndromes that may involve hematologic, endocrine, neuromuscular, musculoskeletal, or cardiac systems. These syndromes may have puzzling or unusual presentations. Small cell carcinomas generally originate in the hilar areas, but a few present in the lung periphery. Small cell carcinomas are among the fastest growing of all of the bronchogenic carcinomas.

Large cell carcinomas make up about $10 \%$ of bronchogenic carcinomas, and they generally present as fairly large peripheral lesions. Because the large cell carcinomas do not share common histologic attributes with any of the other cell types, they tend to form a "wastebasket" category. ${ }^{37}$

\section{Diagnosis}

Presenting symptoms are cough, weight loss, breathlessness, chest pain, and hemoptysis. About three-fourths of patients have cough; two-thirds have weight loss; one-half have breathlessness; one-half have chest pain; and one-third have hemoptysis. There is nothing pathognomonic about the cough or breathlessness of cancer. The more the weight loss, the more ominous the prognosis in terms of time of survival. The most commonly described type of chest pain is a dull episodic aching on the side of the lesion. These pains are more aggravating than severe. Hemoptysis for 3 months suggests a malignancy.

Cigarette smoking is related to all types of bronchogenic carcinoma except for bronchioloalveolar carcinoma in women. So long as cigarette smoking is popular, lung cancer will be common. Fortunately, prospective studies have shown that quitting smoking cuts down on the occurrence of lung cancer. Other causes of lung cancer that deserve mention include passive smoking, asbestos, arsenic, chromium, nickel, coal products, chloromethylethers, vinyl chloride, and ionizing radiation. Searching for presymptomatic lung cancers by periodic chest $\mathrm{x}$-ray films or sputum cytologic studies has not reduced lung cancer mortality, and these tests are not used.

Computed tomography (CT) scans have assisted in the diagnosis and management of lung cancer. The CT scan is the best tool for evaluating mediastinal lymph nodes and mapping out the extent of a lung cancer. ${ }^{38}$ Magnetic resonance imaging allows sagittal and coronal images, and it differentiates vessels from mediastinal masses better than computed tomography. ${ }^{39}$ With these excellent visualizations, particularly with chest CT scans, skinny needle samplings of lung masses and infiltrates have proved reliable and generally accurate in determining the tissue type of abnormal masses.

The key considerations in lung cancer are the tissue type (small cell or other) and the resectability. The presence of lung cancer can be determined by cytology of expectorated phlegm ( $80 \%$ yield but often unclear tissue type); bronchoscopy with biopsy, brush, or lavage ( $80 \%$ yield with clear tissue type); skinny needle aspiration under fluoroscopy or CT scan guidance ( $80 \%$ yield with clear tissue type); mediastinoscopy; and open thoracotomy. Lung cancer is staged in a TNM system. T stands for primary tumor extent; $\mathrm{N}$ indicates nodal involvement; $M$ denotes metastases. ${ }^{40}$ 


\section{Treatment}

The treatment of lung cancer is discussed in Chapter 34.

\section{Pulmonary Embolism}

Rudolf Virchow (1821-1902) was the first to describe pulmonary embolism. He recognized that venous thrombi were the result of phlebitis, and that these thrombi could embolize to the lungs. There are 600,000 cases of pulmonary embolism per year in the United States, and with untreated cases the mortality may be as high as $30 \% .^{41}$ Most pulmonary emboli come from proximal deep thigh venous thrombosis. If the thrombus in the legs is confined to the calf without proximal extension, there is a low risk of pulmonary emboli. Other sites of origin are the pelvic veins, especially after pelvic surgery, infections, and birth; internal jugular veins, especially with the use of indwelling central lines and with head and neck surgery or infections; and the right atrium after atrial fibrillation. The risk of pulmonary embolism is also increased with oral contraceptives, carcinoma of the pancreas, and primary polycythemia. With surgery or acute medical illness, bed rest may result in about a $30 \%$ occurrence of lower-extremity deep venous thrombosis. Venous thrombosis and pulmonary emboli tend to recur.

The patient with a silent deep venous thrombosis of the lower extremities has no complaints but is at risk for pulmonary emboli if the thrombus extends above the knee. If thrombophlebitis is present, the cardinal signs of inflammation may be found, including heat, redness, swelling, and pain. It is difficult to walk on a thrombophlebitic leg. The patient with pulmonary embolism has a wide spectrum of presentations. Most patients have chest pain, either pleuritic or nonpleuritic, breathlessness, fever, and cough. A few have hemoptysis. The signs of pulmonary embolism are variable; they may suggest but not make the diagnosis. Syncope, an ominous indication of massive pulmonary embolism, occurs in $10 \%$ of patients with pulmonary embolism. Interestingly, only about one-third of patients with clinical pulmonary embolism have evidence of phlebitis. ${ }^{42}$

\section{Laboratory Studies}

On the basis of signs and symptoms, it is difficult to make the diagnosis of pulmonary embolism, as these findings are not pathognomonic, and they can be found in chest infections. For example, hypoxemia is found in about $80 \%$ of patients with pulmonary embolism. Objective tests are needed, and pulmonary angiography is the "'gold standard." However, in a suggestive, high risk situation, a perfusion-ventilation scan may suffice for the diagnosis of pulmonary embolism. A normal ventilation scan accompanied by a perfusion scan showing large segmental perfusion defects makes a diagnosis of pulmonary embolism likely. Clinical judgment is needed as to when the ventilation-perfusion scans suffice to rule in or rule out pulmonary embolism and when one proceeds to pulmonary angiography, as there are patients who are not candidates for pulmonary angiography, particularly those with pulmonary hypertension.

There are several tests for detection of deep venous thrombosis of the lower extremities, including impedance plethysmography, Doppler ultrasound, radioactive-labeled fibrinogen testing, and venography. Impedance plethysmography is ef- fective and noninvasive and therefore preferred for diagnosis and follow-up. ${ }^{43}$ Unfortunately, it is not widely available, so that venography is often utilized.

\section{Treatment}

The medical treatment of pulmonary embolism is either anticoagulation or thrombolytic therapy. When comparing these two medical approaches, there is no significant difference in the recurrence rates of pulmonary embolism or in the number of deaths. The thrombolytic therapy accelerates the disappearance of pulmonary emboli and improves pulmonary perfusion with a quicker return of pulmonary artery pressures toward normal. ${ }^{44}$ Probably because more bleeding occurs with thrombolytic therapy (about half of the time) than with heparin therapy (about one-fourth of the time), heparinization is usually used in the treatment of uncomplicated pulmonary embolism. If massive embolism is suspected, as would be the case with shock or syncope, streptokinase thrombolytic therapy is favored, to be followed by heparin. Intermittent intravenous heparin or large-dose frequent subcutaneous heparin can be used. Either the activated clotting time or the partial thromboplastin time is used to monitor heparin therapy. Both are kept at approximately 1.5 to 2.0 times normal, watching for the complications of bleeding. Monitoring the heparin dose is important to cut down the incidence of bleeding as a complication of heparin therapy.

Oral anticoagulant in the form of warfarin is usually started along with heparin, so that both are given over approximately one week; then the heparin is stopped and the oral anticoagulant continued, aiming for a prothrombin time of 1.2 to 1.5 times control values. It takes approximately a week to bring factors II, VII, IX, and X into the therapeutic range of anticoagulation, so there should be a week of overlapping therapy. The clinician must keep in mind the many drugs that interact with warfarin. There is increased sensitivity to warfarin if the patient is taking aspirin, nonsteroidal antiinflammatory drugs, phenytoin, oral hypoglycemic agents, estrogens, anabolic steroids, erythromycin, cimetidine, sulfamethoxazole-trimethoprim, or quinidine. There is decreased sensitivity to warfarin with the concomitant administration of nafcillin, rifampin, griseofulvin, barbiturates, and cholestyramine resins. Warfarin is contraindicated during pregnancy, and dose-adjusted subcutaneous heparin (generally 10,000 units b.i.d.) is a reasonable substitute. Warfarin, of course, cannot be used if there is any significant bleeding problem. ${ }^{45}$ It does decrease the chance of recurrent deep venous thrombosis and recurrent pulmonary embolism, and in uncomplicated cases it is continued for 6 weeks. ${ }^{46}$ If the risk of deep venous thrombotic or pulmonary embolic disease remains high, anticoagulation may be continued on a long-term basis.

Pulmonary emboli and deep venous thrombosis are serious postoperative complications. Heparin 5,000 units subcutaneously 2 hours preoperatively and every 8 hours thereafter for 7 days reduces by two-thirds the occurrence of deep venous thrombosis as measured by the radioactive fibrinogen test and the appearance of pulmonary emboli. ${ }^{47}$

\section{Tuberculosis}

Pulmonary tuberculosis was the most common cause of death in the United States until World War I. Like many infectious 
diseases, its mortality rate began to fall before the antibiotic era. The author's supposition is that this phenomenon may be related to availability of separate bedrooms for children. Infection is spread by microscopic droplets ( 1 to $5 \mu \mathrm{m}$ ) that are aerosolized by coughing or talking. Mycobacteria are killed by ultraviolet light, so sharing a darkened bedroom or other closed area (e.g., a nursing home or jail) is an ideal way to spread the bacillus.

Tuberculosis has been found in Egyptian mummies dating back to $3700 \mathrm{BC}$. The incidence started to climb steeply when urbanization began during the late Middle Ages. By the eighteenth and nineteenth centuries it had become a frequent cause of death. The names of those who succumbed to it read like a Who's Who of Western civilization: Laennec, Mozart, Keats, Nietzsche, and even Eleanor Roosevelt. Tuberculosis incidence in the United States has decreased from 80 per 100,000 in 1930 to about 10 per 100,000 today. Thus the busy family physician encounters, on the average, about one case every 5 years. If one practices in the underdeveloped world, however, it is a different story, as there it may still be the leading cause of death.

\section{Pathology}

Tuberculosis is caused by a small bacterium with a thick lipid wall, making it particularly resistant to destruction from either macrophages or drugs. It multiplies slowly and can lay dormant for long periods of time. The infection usually starts after the patient has inhaled infected droplets that lodge in the periphery of the lung, where they cause a local inflammatory reaction. There is usually hematogenous spread; lymph nodes, other areas in the lung, kidneys, liver, and bone are the most common organs seeded. Clinical disease rarely occurs at this stage, although occasionally foci may irritate or rupture into the pleura causing a pleural effusion. (An old adage, and still a wise one, is that one must rule out tuberculosis in an unexplained pleural effusion in a young person.) After the initial asymptomatic infection, most individuals are left only with a positive skin test and possibly a parenchymal calcification (Ghon's lesion), which is often associated with calcified hilar lymph nodes (Ranke complex).

\section{Epidemiology}

In the United States more than $90 \%$ of active tuberculosis is actually reactivation of an old infection, i.e., breakdown of the original foci years later. Why it recurs is poorly understood. Risk factors for active tuberculosis include recent weight loss (associated with malnutrition or alcoholism), poorly controlled diabetes, and immunosuppression (from steroids, AIDS, or cancer). The Holmes-Rahe Life Change Index, now frequently used to measure stressful life events, was first developed to help predict the development of active tuberculosis.

Of those infected with bacilli (i.e., converting their skin test), it is estimated that only 2 to $3 \%$ develop active tuberculosis within the first year after exposure. The risk is higher in infants and adolescents. In all, this risk falls to a baseline rate of reactivation variously estimated at 1 per 1,500 to 1 per 4,000 per year. Of all those with positive skin tests, no excess risk factors, a normal chest $\mathrm{x}$-ray film, and not thought to be recent converters, one person of approximately $2,000 \mathrm{de}$ - velops active tuberculosis each year. Over a lifetime, then, most people who convert their skin tests run a 2 to $3 \%$ chance of developing the disease initially and another 2 to $3 \%$ chance over the rest of their lives. As there are few recent converters in the United States, most active tuberculosis comes from the previously infected group.

\section{Diagnosis}

Symptoms of pulmonary tuberculosis are cough, hemoptysis, weight loss, fatigue, and low grade fevers (often manifested by night sweats). Some persons are asymptomatic, and the diagnosis is made by a chest $\mathrm{x}$-ray film obtained for some other purpose or on routine skin testing. This study most frequently shows a fibronodular upper lobe infiltrate. Cavities may form; and hilar node adenopathy, pleural effusion, or upper lobe volume loss may be present. The skin test is positive in more than $90 \%$ of patients but may be negative in the elderly or immunosuppressed. A positive skin test alone implies only that someone has been infected at some time in the past; it tells nothing about current activity. The definite diagnosis is based on a smear and culture of the sputum or other infected material. Smears may be prepared using a Ziehl-Nielsen technique that causes the organisms to appear red; more frequently a fluorescence technique is used. If the patient cannot cough up a specimen, obtaining aerosol-induced sputum is tried. Gastric washings are rarely done. In tuberculous pleural effusions, the fluid is usually a mononuclear exudate. Smear and culture are usually negative. Diagnosis is based on seeing caseating granulomas in the pleural biopsy.

The sputum smear may be negative in active disease. Culture incubation takes 6 to 8 weeks, although shorter culture techniques are being developed. Sensitivities to antituberculosis drugs may be reported then (direct sensitivities) or sometime thereafter (indirect sensitivities). Newer diagnostic techniques, including serologic tests and more rapid growing laboratory media, are under development.

\section{Therapy}

The current recommendation for antituberculosis therapy for active disease is isoniazid (INH) $300 \mathrm{mg}$ q.d. and rifampin 600 $\mathrm{mg}$ q.d. for 9 months. There are many appropriate variations. If the individual has had previous therapy or comes from Central America or Southeast Asia, there is a higher probability of drug resistance. In this instance, therapy consisting of three drugs is used, adding ethambutol at 15 to $25 \mathrm{mg} / \mathrm{kg}$ until the drug sensitivities are back. If at that time the organism is INHresistant, rifampin and ethambutol are continued for 18 months; if the organism is rifampin-resistant, INH and ethambutol are used for 18 months.

A six-month course of chemotherapy can be used. Acceptable results are achieved using rifampin $600 \mathrm{mg}$ q.d., isoniazid $300 \mathrm{mg}$ q.d., ethambutol 15 to $25 \mathrm{mg} / \mathrm{kg}$ q.d., and pyrazinamide 15 to $30 \mathrm{mg} / \mathrm{kg}$ (up to $2 \mathrm{~g}$ ) q.d. or streptomycin for 2 months, and then continuing INH and rifampin for an additional 4 months. See Table 17.6 for dosage and common side effects of these agents. Regimens using drugs for fewer than 6 months are not acceptable because of high rates of failure and relapse. Infants and children are treated with a 9-month regimen, as are those with extrapulmonary tuberculosis.

In 1982, of all reported tuberculosis cases in the United 
Table 17.6. Therapy for tuberculosis

\begin{tabular}{clc}
\hline \multicolumn{1}{c}{ Drug } & Usual adult daily dosage & \multicolumn{1}{c}{ Common side effects } \\
\hline $\begin{array}{c}\text { Primary } \\
\text { Isoniazid }\end{array}$ & $300 \mathrm{mg}$ & $\begin{array}{c}\text { Peripheral neuritis (use vitamin } \mathrm{B}_{6} 25 \\
\text { mg q.d.); hepatitis (see text); in- } \\
\text { creases Dilantin level; CNS reac- } \\
\text { tions } \\
\text { Colors urine and stool orange; accel- } \\
\text { erates metabolism of certain drugs } \\
\text { (birth control pills, Coumadin, } \\
\text { methadone); thrombocytopenia; } \\
\text { hepatitis; febrile reactions }\end{array}$ \\
$\begin{array}{c}\text { Secondary } \\
\text { Ethambutol }\end{array}$ & $15-25 \mathrm{mg} / \mathrm{kg}$ & $\begin{array}{c}\text { Optic reactions (rare at dose of 15 } \\
\text { mg/kg); skin rash }\end{array}$ \\
Pyrazinamide & $15-30 \mathrm{mg} / \mathrm{kg}$ (max. 2 g) & $\begin{array}{c}\text { Hepatitis; hyperuricemia; gastrointes- } \\
\text { tinal disturbance }\end{array}$ \\
Streptomycin & $0.75-1.00 \mathrm{~g}$ IM q.d. & $\begin{array}{c}\text { Eighth nerve damage: (follow audio- } \\
\text { gram); nephrotoxicity; skin rash }\end{array}$ \\
\hline
\end{tabular}

States, $16 \%$ were extrapulmonary. The most common sites are kidneys and bone, but any organ can be infected. ${ }^{48}$ Systematic follow-up after treatment is not indicated unless there is reason to doubt compliance.

\section{Preventive Treatment}

Because active pulmonary tuberculosis is a result of an infection that took place months to years before, it affords the opportunity for prevention. Based on trials in Alaskan Eskimos, there is evidence that more than $90 \%$ efficacy in preventing future active disease can be achieved with INH chemoprophylaxis. The indications for use of chemoprophylaxis include ${ }^{49}$ :

1. Household members and other close contacts of newly diagnosed, sputum-positive (infectious) patients

2. Newly infected persons, e.g., conversion of the skin test within a 2-year period

3. Significant tuberculin skin test reactors with an abnormal chart x-ray film (more than a calcified node or pleural thickening)

4. Tuberculin reactors in special clinical situations such as those on steroids ( $>15 \mathrm{mg}$ of prednisone q.d.), silicosis, certain cancers, chronic renal insufficiency, poorly controlled diabetes, and conditions associated with nutritional deficiency and weight loss, including gastrectomy and intestinal bypass

5. Tuberculin-positive persons, age 35 and under, with no other risk factors

The last group is by far the largest, and controversy exists regarding whether all (particularly those over age 20 who have normal chest x-ray films and are not recent converters) should be treated. Risk-benefit analysis favors treating all persons up to age 35 . In those with normal chest $x$-ray films, 6 to 9 months of INH at $300 \mathrm{mg}$ q.d. is adequate. A 9- to 12-month treatment period is indicated for those with abnormal (but presumably stable) chest $\mathrm{X}$-ray films.
The major concern about INH is drug-induced hepatitis. A large United States Public Health Service (USPHS) study showed the overall incidence to be about $1 \%$. In persons under age 20 hepatitis was extremely rare, whereas in those over age 50 it occurred in $2 \%$. There are usually prodromal symptoms, e.g., fatigue and anorexia, which may occur weeks to months before clinical toxicity; $70 \%$ of hepatitis occurs during the first 3 months of therapy. The best way to follow patients on INH is regularly (monthly), asking about symptoms of hepatitis. Biochemical monitoring of those over age 35, heavy drinkers, and those with other liver disease (e.g., chronic hepatitis) is reasonable. Up to $10 \%$ may show a mild, asymptomatic rise in enzymes (less than three times normal). In these individuals INH does not need to be discontinued, but they should be followed more closely.

\section{Interstitial Lung Diseases}

Interstitial lung diseases are increasingly common, affecting more than 10 million Americans. A wide variety of insults lead to similar patterns of either interstitial or alveolar inflammation and fibrosis. Patients with these diseases generally present with breathlessness, first with exercise, and finally at rest. The shorter the period of breathlessness before treatment (e.g., 1 year or less), the better the prognosis with treatment. Often there is a concomitant nonproductive cough, and there may be systemic symptoms such as fever and weight loss. The physical examinations of these patients are likely to show bibasilar crepitations. There may or may not be cyanosis and digital clubbing. In advanced cases the findings of cor pulmonale are prominent.

The chest $\mathrm{x}$-ray shows a diffuse haziness or finely reticulated appearance at the lung bases. As the process becomes more fibrotic, a honeycomb appearance can be noted on the $\mathrm{x}$ ray film. Pulmonary function tests show restrictive patterns with decreased lung volumes and decreased carbon monoxide diffusion capacities. The $\mathrm{FEV}_{1}$ value as a percent of the 
forced vital capacity is usually normal. Arterial blood gases show hypoxemia.

A variety of insults can produce the same clinical, roentgenographic, and laboratory abnormalities. First, there are more than 30 inorganic dusts capable of producing interstitial fibrosis. Coal, silica, and asbestos are the most common causes. The collagen vascular diseases, e.g., rheumatoid arthritis, may present as interstitial lung disease. A wide variety of infections can produce interstitial lung disease, particularly cytomegalovirus, the influenza viruses, Pneumocystis disease, and fungal disease. Hypersensitivity pneumonias may result from bird proteins and molds from compost, dust, bark, cheese, and wheat, leading to lung scarring. Certain drugs, e.g., amiodarone, nitrofurantoin, hydralazine, and procainamide, produce a similar pattern of immunologic lung damage with interstitial disease. Excessive radiation can produce this same pattern. Sarcoid, a disease of unknown etiology, is a frequent cause..$^{50}$

The presence of interstitial lung disease can be diagnosed by the clinical history, physical examination, chest x-ray film, and pulmonary function testing. The problem is to identify which of the many etiologic agents is responsible. Although gallium scans and bronchoalveolar lavage can give helpful information in terms of the activity of the disease process, they do not predict the natural history, and they are not currently relied on regarding treatment. A tissue diagnosis is required; and if a transbronchial biopsy is negative or shows only nonspecific inflammation, an open lung biopsy should follow. If the biopsy shows a prominent acute cellular infiltrate without end-stage fibrosis, corticosteroid therapy is generally indicated. If possible, avoiding the offending agent is the first step (as with a hypersensitivity pneumonitis). Next is the suppression of inflammation, and corticosteroids are generally the agents of choice. The patient's symptoms, physical examination, chest $\mathrm{x}$-ray film, and pulmonary function tests, especially lung volumes and carbon monoxide diffusion capacity, are followed to ascertain the response. Steroids are generally started at $1 \mathrm{mg} / \mathrm{kg}$ q.d. for several months and then tapered to 10 to $15 \mathrm{mg}$ q.d. for upward of 3 months to a yearor years, depending on the diagnosis and responsiveness to therapy. The task is to turn off the interstitial and alveolar inflammatory processes so as to preserve lung function. If interstitial lung disease is progressive and unresponsive to corticosteroid or other treatments, the natural history is usually one of death due to respiratory insufficiency within 2 to 4 years. ${ }^{51}$

\section{Bronchiectasis}

Bronchiectasis means abnormal dilation of the bronchi. Its prevalence is probably about $0.5 \%$ and decreasing. It is usually the result of a severe episode of pneumonia during childhood. Most frequently, it is from a viral pneumonia, but it may be from bacterial, mycoplasmal, tuberculous, fungal, or aspiration pneumonia. Foreign body aspiration, a chemical burn, or a congenital defect also can cause bronchiectasis.

The pathology can be classified into two types: (1) Cylindral bronchietasis. Here there is a loss of tapering of the bronchi but with a smooth outline. This type is often transient and reversible, and may follow as many as $40 \%$ of pneumonias. (2)
Saccular bronchiectasis. There is more extensive irreversible damage to cartilage and smooth muscle with this type, and "sacs" are seen on bronchograms. In either case, the mechanism of mucociliary clearance is damaged. The left lower lobe is most frequently involved, probably because of the smaller bronchi. One or more lobes may be involved.

The history is one of chronic productive cough, usually purulent and foul-smelling. There are often febrile exacerbations. The cough may be worse in the morning, and for some reason recurrent sinusitis is common. Hemoptysis may be a complicating factor and can be severe. If the disease progresses, dyspnea may develop.

On physical examination the most common findings are rattles and crepitations, even when the patient is asymptomatic. The chest x-ray film is usually abnormal but nondiagnostic, with increased lung markings, honeycombing, and atelectasis. Pulmonary function tests show small airway disease with mild obstructive defects. The CT scan is usually not helpful.

Additional work-up might include looking for cystic fibrosis or $\alpha_{1}$-antitrypsin deficiency as well as sputum cultures for tuberculosis and fungi. Sputum cultures for bacteria usually reveal a mixed flora. Bronchography is indicated only if surgery is being considered.

Treatment usually consists in broad-spectrum antibiotics to help clear purulent sputum, bronchodilators if there is a reversible component on pulmonary function testing, and chest physiotherapy. The latter is usually done with inhaled bronchodilators followed by postural drainage and chest percussion two to four times a day. It does not change pulmonary functions, but it does help in the mobilization of sputum. Bronchiectasis is the one pulmonary disease where patients benefit from physical therapy. ${ }^{52}$

If obstruction from a foreign body or tumor is suspected, bronchoscopy is indicated. If the patient cannot be adequately controlled on medical therapy after more than 12 months, surgery to remove the affected lobe(s) may be considered. Surgery is often curative if the disease is in one or two contiguous lobes only. The long-term prognosis for most patients is good with relatively stable pulmonary function. ${ }^{53}$ Patients should receive pneumococcal vaccine plus yearly flu vaccine.

\section{Bronchiolitis}

Bronchiolitis is an acute viral respiratory disease of infants and young children. Its incidence may be as high as 5\% in children, usually in those under age 2 . It most typically occurs during winter and early spring in 3- to 6-month-old male infants. The cause is most commonly respiratory syncytial virus (RSV) (which is contagious within schools and families), although other viruses such as the parainfluenza virus may also cause bronchiolitis. In older children and adults, RSV produces bronchitis or upper respiratory tract infection (URI). The virus causes an inflammation and obstruction of the small bronchioles.

The typical clinical picture starts with a mild upper respiratory infection and cough. Within 1 to 2 days after onset, tachypnea develops. Respirations are rapid and shallow, with overexpansion of the chest and prolonged expiration. The infant may be unable to suck and can become dehydrated. Fever, if present, is low grade. Coryza, otitis, and pharyngitis may be 
present. Physical findings include intercostal retractions and nasal flaring. Wheezes and crepitations are usually present, and cyanosis may develop. The white blood cell count is normal, and the chest x-ray film usually shows hyperinflation (e.g., lowered diaphragms and hyperlucency). Patchy perihilar infiltrates, atelectasis, or even pneumonic changes may be present. Blood gases show hypoxia. The differential diagnosis includes foreign body obstruction, pertussis, or bronchopneumonia. Asthma is rare in children under the age of 12 months but obviously is in the differential diagnosis.

The usual course is a worsening condition for 3 to 4 days and then gradual resolution over 1 to 2 weeks. Ambulatory treatment is supportive with careful attention to fluid status and respiratory fatigue.

Hospitalization may be required for hypoxemia or dehydration. It is recommended in infants under age 3 months if there is cyanosis or a history of apnea, if the respiratory rate is more than 60 per minute, if the arterial $\mathrm{PCO}_{2}$ is $>45 \mathrm{~mm} \mathrm{Hg}$, or if the arterial $\mathrm{Po}_{2}$ is $<60 \mathrm{~mm} \mathrm{Hg}$ on room air. Treatment is with oxygenation, mist, and mechanical suction of the upper airways. Pneumonia and respiratory failure requiring ventilatory assistance occasionally develop. Continuous positive airway pressure is usually effective in the management of respiratory insufficiency. Bronchodilators or steroids have not been shown to benefit. ${ }^{54}$ If asthma is a strong consideration in the older infant, a trial of inhaled epinephrine or beta- 2 agonists may be considered. Ribavirin, a synthetic nucleoside given by inhalation, may also be considered in those seriously ill. 55

In infants with a difficult course, cystic fibrosis, cardiac lesions, or pulmonary congenital anomalies are kept in mind as underlying conditions. Many infants subsequently have recurrent wheezing in association with viral illness, as well as an increased risk of asthma. The long-term prognosis is excellent, but subtle abnormalities of pulmonary function are seen in many children up to 10 years after bronchiolitis. ${ }^{56}$

\section{Fungal Diseases of the Lung}

Although serious fungal infections of the lung are relatively uncommon in many parts of the United States, they are seen enough to warrant inclusion in this chapter. The following is a brief discussion of the common presenting symptoms of acute, disseminated, and chronic (progressive) disease, diagnostic tests, and therapy of pulmonary fungal infections.

\section{Coccidioidomycosis}

Coccidioidomycosis is caused by a fungus found in the soil of semiarid areas of the Southwest. The primary acute infection is asymptomatic in more than $60 \%$ of infected individuals. The rest have variable symptoms, including cough, dyspnea, fever, rash, pharyngitis, arthralgias, and erythema nodosum. About 5\% develop persistent or progressive pulmonary infection. Immunocompromised individuals, Blacks, Filipinos, and Native Americans are at high risk. Chest x-ray films usually show nondiagnostic and transient infiltrates. About $20 \%$ have hilar adenopathy, and as many as $10 \%$ may have a small pleural effusion. In those with progressive pulmonary disease, the pulmonary infiltrate or adenopathy lasts more than 6 weeks. There may be multiple pulmonary nodules, thin-walled cavi- ties, or progressive pneumonia. The organism is usually present in the sputum. The diagnosis is made on the basis of a characteristic exposure and history, along with positive skin tests, sputum smears, and positive serology. Two skin tests are available: spherulin, which is the more sensitive test, and coccidioidin, which is the more specific test. Early in the course of the disease, the latex agglutination and precipitin serology are positive. Later in the course, complement fixation and immunodiffusion tests become positive. Patients with high complement fixation titers have a greater risk of progressive pulmonary disease. Rarely, coccidioidomycosis can disseminate, with infections of the soft tissue, skin, meninges, or bone.

The question of drug therapy is often a difficult one. Most infections with coccidioidomycosis are self-limited. The decision to treat depends on the risk, the extent of the disease, and the symptoms. In those with progressive disease and pulmonary cavities, ketoconazole is the drug of choice. For those with progressive pulmonary pneumonia or dissemination, ketoconazole, amphotericin $\mathrm{B}$, or surgery may be needed..$^{57.58}$

\section{Histoplasmosis}

Histoplasmosis is caused by a soil fungus. The fungus is found in bird droppings and moisture in the southeastern and central United States. Infection is common (although usually asymptomatic). In some areas of the Southeast as many as $90 \%$ of the population may have positive skin tests. Three clinical syndromes are described.

With a very heavy inoculum of spores, one may develop acute histoplasmosis, with a flu-like syndrome and symptoms of fever, cough, dyspnea, chest pain, arthralgias, and possibly erythema nodusum. The chest $\mathrm{x}$-ray film may show bilateral pneumonia (usually in the lower lobes) and occasionally hilar adenopathy or a small pleural effusion. The sputum cultures are usually negative, but complement fixation titers are elevated. A fourfold rise in the titer or a single titer that is $\geq 1: 32$ is highly suggestive of acute histoplasmosis. The skin test is positive after 2 to 3 weeks, but skin tests may cause a rise in titers, so they are usually withheld until after blood for serology is obtained.

The treatment is supportive. When healed, on chest $\mathrm{x}$-ray film there may be a characteristic peripheral pulmonary nodule with concentric calcifications. Splenic calcifications are common. Occasionally calcified hilar or paratracheal lymph nodes produce entrapment syndromes of the right middle lobe, trachea, superior vena cava, or esophagus.

Rarely, histoplasmosis disseminates in immunocompromised men or infants. It can affect multiple organs: liver, gastrointestinal tract, bone marrow, and adrenals. The course may be fulminant, particularly in infants. The diagnosis requires a positive culture, usually from a biopsy. Treatment is with amphotericin B.

Chronic pulmonary histoplasmosis is a disease of middleaged men with COPD. It probably results from reinfection. There can be mild symptoms, with low grade fever, malaise, weight loss, and productive cough. On chest $\mathrm{x}$-ray film, cavities may be seen that appear much like those caused by tuberculosis. Treatment is ketoconazole if there is cavitary disease. ${ }^{59,60}$ 


\section{Blastomycosis}

Blastomycosis is an uncommon pulmonary disease, most frequently of middle-aged men in the southeastern or central United States. The fungus is found in soil, but how it infects humans is unclear. Outdoor vocations and avocations seem to increase the risk of infection. As with other fungal diseases, there is a wide range of pulmonary symptoms. Most are mild or asymptomatic and heal spontaneously. There are two clinical syndromes that are caused by the fungus.

1. Acute pneumonitis is a "flu-like" syndrome characterized by productive cough, fever, chest pain, and myalgias. As with other fungal infections, the disease is usually self-limited. Occasionally, dissemination occurs.

2. Chronic pulmonary disease may be confused with tuberculosis or histoplasmosis. With this chronic disease as many as $70 \%$ of the patients have dissemination. The skin is the most common site, with small nodules that can ulcerate. Bones, soft tissue, and the genitourinary tract can also be involved. The chest $\mathrm{x}$-ray findings are variable and nonspecific, showing infiltrates, adenopathy, mass lesions, or occasional cavities. The definitive diagnosis is based on demonstration of the fungus microscopically or in culture from the sputum or lesions. (The culture takes 1 to 3 weeks.) Skin tests and serology are not helpful. The decision on whether to treat is a clinical one. One might use ketoconazole if the disorder is not severe or amphotericin B for severe chronic disseminated infection. ${ }^{61,62}$

\section{Aspergillosis}

Aspergillosis is a fungus that is ubiquitous worldwide and fortunately rarely causes disease in normal hosts. Three clinical syndromes are described. First is the aspergilloma, which is a mass of mycelial elements, fibrin, and cellular debris that forms within a preexisting cavity (usually from tuberculosis, other fungal diseases, sarcoid, or pneumoconiosis). The presenting symptom in more than $50 \%$ of cases is hemoptysis. The chest $\mathrm{x}$-ray film is characteristic, usually showing an upper lobe cavity with a shadow inside. Serum precipitating antibodies are present and Aspergillus organisms can be grown from sputum or surgically removed material. The treatment is controversial: There are no clear-cut guidelines governing the decision whether to leave the patient alone, perform surgery, use steroids, or use intracavity or systemic antifungal agents. Usually there is a relatively benign course, although the underlying lung disease may be severe.

A second manifestation is allergic bronchopulmonary aspergillosis (ABPA). ABPA is a hypersensitivity disease, often seen in patients with long-standing asthma. Presenting symptoms are usually cough, productive of mucous plugs, hemoptysis, and chronic wheezing. There may be recurrent fevers and pneumonia symptoms. The chest $\mathrm{x}$-ray film shows changing pulmonary infiltrates, most commonly in the upper lobes. The serum shows elevated immunoglobulin E (IgE) levels and specific precipitating antibodies. Skin tests show immediate and delayed hypersensitivity to Aspergillus antigen. Expectorated sputum shows mucous plugs with mycelia. Sputum culture is usually but not always positive. There may be laboratory contamination, and thus diagnosis rests on positive skin tests and serum precipitins. Treatment is aimed toward control of bron- chial spasm with bronchodilators, cromolyn, and inhaled steroids. Systemic steroids may be required for control and are used for exacerbations, as the disease often has remissions and recurrences. ${ }^{63}$

Lastly, invasive aspergillosis can be seen in the immunocompromised individual. Clinically there is dyspnea, cough, fever, and chest pain. There may also be a chronic necrotizing pulmonary disease, with infiltrates and cavities. The diagnosis is usually by lung biopsy, as it is clinically difficult to distinguish it from Pneumocystis or other fungal pneumonias. Prognosis is poor in the immunocompromised patient, unless one is able to make the diagnosis early and modify the underlying immune status. ${ }^{64}$

\section{Cryptococcosis}

Cryptococcus is a ubiquitous fungus whose main reservoir seems to be birds (primarily pigeons). There is a wide spectrum of the disease, with most infections probably being asymptomatic. The true spectrum of cryptococcal infection is difficult to judge because there is no specific serologic or skin test. Symptoms are usually cough, chest pain, and low grade fever. Chest X-ray films usually show a peripheral, lower lobe, mass-like lesion. The lesion may cavitate or may appear as multiple nodules or an infiltrate. In the immunosuppressed patient there is a significant risk of dissemination. Meningeal disease particularly has a poor prognosis, so it is recommended that the cerebrospinal fluid (CSF) be examined for lymphocytes, increased protein, cryptococcal antigen, and fungus (India ink preparation and culture) in those who have cryptococcal lung disease. As with other fungal diseases, indications for treatment with pulmonary disease are not well defined. In a normal host with only pulmonary disease, supportive treatment is warranted. However, treatment of disseminated disease is amphotericin B and flucytosine or ketoconazole. ${ }^{65}$

\section{Nocardiosis}

Nocardia is not a true fungus but a soil bacterium that can develop mycelia. It is gram-positive and acid-fast. The disease usually occurs in men and often (but not always) in those who are immunosuppressed. Most patients have only a pulmonary infection, but the infection can disseminate. Symptoms are productive cough, chest pain, and low grade fevers. On chest $\mathrm{x}$-ray film there is nodular disease, cavities, or abscesses, which can form sinus tracts through the chest wall. Extrapulmonary disease can affect the skin, soft tissue, or central nervous system (brain abscess). The diagnosis depends on isolating the organism from sputum or tissue. There is no serologic or skin test. The treatment is sulfisoxazole, alone or in combination with trimethoprim. The treatment is for prolonged periods (up to 18 months) with high doses initially. Chloramphenicol, tetracycline, and minocycline may also be effective. ${ }^{66}$

\section{Actinomycosis}

Actinomyces is an anaerobic gram-positive bacterium that forms filamentous hyphae. The pulmonary infection is thought to come from organisms residing around carious teeth. The presenting symptoms are the same as those for many fungal 
diseases: productive sputum, fever, and chest pain. The chest $\mathrm{x}$-ray film may appear similar to that seen with pneumonia, or there may be a large mass that can extend to the chest wall and cause empyema. The diagnosis depends on the demonstration of organisms in tissue. Sulfur granules (1- to 2-mm clumps of mycelia) are sometimes seen in the sputum or in material from a chest wall sinus tract. Treatment is penicillin in high doses for long periods of time (up to 18 months). Tetracycline and clindamycin may also be effective.

\section{Sarcoidosis}

Sarcoidosis is a multisystem granulomatous disorder of unknown cause characterized by an enhanced cellular immune process at sites of involvement. Sarcoidosis is more prominent in Scandinavia and temperate climate areas. ${ }^{67}$ Sarcoidosis usually involves the lungs, skin, eyes, liver, spleen, parotid glands, phalangeal bones, muscles, heart, and nervous system. Most cases remit spontaneously, but some have a chronic course, and a few end in death. The cause(s) of sarcoidosis is unknown.

Sarcoidosis presents many immunologic abnormalities. The number of circulating $\mathrm{T}$-cells is reduced, resulting in an impaired cellular response. Bronchoalveolar lavage reveals an increased number of T-cells with the inference that the lung is directly involved in an immunologic reaction. ${ }^{68}$ Whereas cellular immunity appears depressed systemically, humoral immune markers are increased, as frequently there are higher titers of common antibodies and an increase in antinuclear antibodies, rheumatoid factor, and immunoglobulins.

With active sarcoidosis, biopsies of the salivary glands are positive in about one-third of patients; liver biopsies are positive in approximately 75\%; and mediastinoscopy and open lung biopsies have almost $100 \%$ positive results. Bronchial and transbronchial lung biopsies are now generally the biopsies of choice in the work-up of a patient with sarcoidosis. They are positive about $80 \%$ of the time, and in general (save for about a $1 \%$ incidence of pneumothorax), they are considered safe. 69

For a clinician, sarcoid is one of the most fascinating diseases to be diagnosed. Patients generally present with nonspecific systemic complaints including weight loss, fatigue, fever, and weakness. Cough is a frequent symptom, and the physical examination most commonly shows enlarged lymph glands. Less frequently there are striking manifestations such as erythema nodosum. Sarcoidosis has multiple presentations, including, basilar meningitis, complete heart block, iritis, or hepatic granulomas. ${ }^{67}$

Laboratory tests are not specific for the diagnosis of sarcoidosis. The serum angiotensin-converting enzyme is increased in many patients with sarcoidosis, but its level is not an accurate measurement of activity. Positive gallium lung scans are indicators of active disease, but they are not accurate prognosticators. The Kveim test, a subcutaneous granulomatous reaction to injected splenic material from a sarcoid patient, is of historical interest. The chest $x$-ray film is the best indicator of lung involvement. It can range from normal, to large hilar adenopathy (unilateral or bilateral), to infiltrates and fibrosis.

If the course seems benign, drug treatment is avoided and should not be prescribed for minor symptoms and signs. Active pulmonary disease with marked impairment is the most common cause for treatment. Other indications include involvement of heart, brain, liver, or eye. Treatment with corticosteroids is usually given in the setting of serious, progressive disease. The decision to treat means starting with 40 to 60 mg of prednisone daily and tapering down slowly over several months to maintenance doses of $10 \mathrm{mg}$ daily or less. Relapses must be treated early and vigorously. The patient can be followed using clinical symptoms, chest x-ray films, and pulmonary function tests, e.g., the carbon monoxide diffusion capacity.

\section{Asbestosis}

Asbestos is a fibrous silicate mineral. It is virtually indestructible and is an excellent insulator. Asbestos is used primarily in the construction industry for cement piping, roofing, paneling, and insulation. In the past it was used in automobile brake shoes and clutches. One can get exposure from mining, industry, or the environment (e.g., laundering exposed clothes or riding dirt bikes where there is asbestos-bearing rock). Asbestos is used in more than 3,000 products. It has been recognized as a health problem in the mining and manufacturing sectors for more than 70 years. Approximately 10 million people worldwide have had significant exposure to asbestos.

Asbestosis refers to an interstitial pulmonary fibrosis that develops 10 to 30 years after exposure. Asbestos fibers set up a fibrous reaction; they are never metabolized. Asbestosis develops in up to $50 \%$ of exposed workers. It is asymptomatic until the fibrosis is enough to cause dyspnea. In patients with symptoms, pulmonary function studies show decreased lung volumes, and the chest x-ray film usually shows interstitial fibrosis. Pleural plaques form in some patients, involving the lower lobe and diaphragmatic parietal pleura. There is no way to predict who will get asbestosis, but it is more likely in workers who were exposed before 1950 or who inhaled significant quantities of visible dust. Treatment is symptomatic. Smoking cessation must be strongly urged.

There is a fivefold increased incidence of lung cancer in asbestos workers, appearing 25 to 40 years after the initial exposure, and the risk is dose-related. Smoking compounds the risk, so that in smokers there is up to a 50-fold increased incidence of lung cancer. Gastrointestinal and laryngeal cancers are also increased. The lung cancer is usually adenocarcinoma and accounts for as many as $20 \%$ of deaths in asbestosexposed individuals.

Mesothelioma was formerly a rare tumor that has an increasing incidence in asbestos-exposured individuals. It can develop after a 30- to 40-year lag following asbestos exposure. It is not related to smoking. It usually presents as a large pleural effusion, and the diagnosis is best made by open pleural biopsy. It has a poor prognosis, but chemotherapy or immunotherapy may prolong survival. ${ }^{70}$

Even brief exposures to asbestos increase the risk of cancer. Therefore any exposure is unsafe. ${ }^{71}$

\section{Sleep Apnea}

Sleep apnea is defined as cessation of air flow at the nose or mouth for more than 15 seconds during sleep. A sleep history 
is part of every thorough work-up. When indicated, inquiry is made regarding daytime sleepiness, the length of sleep at night, if there is difficulty falling asleep or staying asleep, and if there is snoring or night tremors. A sleep diary kept for 1 to 2 weeks is often useful for assessing sleep disorders. ${ }^{72}$

Snoring is the most common sleep disorder: $40 \%$ of men and $28 \%$ of women snore. It is more common in older and overweight individuals. Some but not all individuals who snore develop sleep apnea. Differentiation between snoring and sleep apnea is sometimes indistinct.

Sleep apnea may be divided into central and obstructive types. Almost any central nervous system lesion can cause central apnea, and it is much less common than obstructive apnea. Obstructive apnea is caused by a decreased tone in the upper airway along with continued diaphragmatic and abdominal movements. There is an invagination of the posterior pharyngeal wall. The obstruction can last up to 2 minutes and continues until there is arousal secondary to hypoxia. It can occur as many as 500 times a night.

The individual's bed partner can readily confirm apneic spells and usually describes the person as having intermittent loud snoring or gasping, followed by silence, in which the patient may have increased movements of the thorax and abdomen. The arms or legs may thrash about as well. This respiratory silence is terminated by a loud snore. The thrashing or snoring may cause the bed partner to seek another room for sleep. Because the patient has relative sleep deprivation, there may be increased daytime drowsiness, morning headaches, or increased irritability. The apneic episodes can precipitate significant cardiac arrhythmias. On physical examination the person is usually obese with a short neck and large jowls. Ear, nose, and throat examination reveals a long soft palate and uvula. Hypertension is common in those with sleep apnea; and cor pulmonale or polycythemia may be found in those with severe disease.

Often the diagnosis is made by history, although there are false positives. Sometimes it is necessary to refer the patient to a sleep laboratory for polysomnography. In some communities home monitoring equipment is available that can make the diagnosis. Finally, significant family and marital issues may be present, as snoring and sleep apnea can be disruptive. ${ }^{73,74}$

Treatment consists in life style changes: weight reduction, stopping smoking, no alcohol before bed, and no hypnotics. Heavy snorers may snore in any sleep position. However, sleeping on one's back may cause increased snoring as the soft palate, uvula, and tongue fall back into the airway. Learning to sleep on one's side can help. One way to do this is to sew a tennis ball into a pocket on the back of the nightshirt or pajama top. Others have tried raising the bed in a manner similar to the treatment for hiatal hernia or wearing a whiplash collar at night to keep the chin extended.

If life style changes are not possible or indicated, a tightfitting nasal mask for continuous positive pressure breathing is the best next step. However, the apparatus is cumbersome, and it is often accepted only after a good deal of patient education and training. Three surgical procedures have been used for treatment of obstructive apnea: tracheostomy, uvulopalatopharyngoplasty (to enlarge the airspace of the pharynx), and mandibular osteotomy with hyoid bone advancement. If medical treatment fails, surgery may give excellent results.

\section{Pneumothorax}

Spontaneous pneumothorax refers to air in the pleural space with partial or complete lung collapse. A primary spontaneous pneumothorax is most common in 20- to 30-year-old men, with a male/female ratio of occurrence of approximately 6:1. Causes of secondary spontaneous pneumothoraces include iatrogenic, bullous emphysema, sarcoidosis, asthma, and cystic fibrosis, and rarely they are associated with menses; there is also an association with smoking. Both primary and secondary pneumothoraces have recurrence rates of $50 \%$ in 10 years.

A pneumothorax usually presents with sudden chest pain and breathlessness; these symptoms may resolve within several days, even if the pneumothorax persists. The physical examination reveals decreased to absent breath sounds and increased tympanic percussion. In COPD patients, pneumothorax may be difficult to detect on physical examination. The chest $x$-ray film is the key to diagnosis.

If the pneumothorax is well tolerated, it can be treated expectantly, anticipating a 10-day reabsorption time. If the pneumothorax is compromising ventilation, either a small chest tube with a Heimlich one-way flutter valve or a traditional large chest tube attached to a water seal with suction is needed.

Indications for a formal thoracotomy with definitive pleurodesis to obliterate the pleural space is either an initial pneumothorax that does not stop leaking after 7 days of a chest tube or a third recurrence of either a primary or secondary pneumothorax.

The main life-threatening complication of a pneumothorax is that of a tension pneumothorax. Its presence must be suspected if there is catastrophic breathlessness, absent breath sounds, or a shift of the trachea. Immediate decompression is needed, even before chest x-ray films are obtained. Placement of a needle over an upper anterior rib into the pleural space relieves the tension, and a diagnostic hissing is heard. A chest tube is then placed. ${ }^{75}$

\section{Rib Fractures}

Rib fractures are common. Diagnosis is made by history and physical examination. The patient may have heard a snapping sound in the chest with a cough, trauma, or a particular maneuver. Point tenderness exists over the fracture site. Chest $\mathrm{x}$ ray films may not show the fracture. Rib fracture is a clinical diagnosis.

The treatment is simply relief of pain, generally with acetaminophen and codeine, and the avoidance of painful postures and maneuvers. Splinting of the chest is usually not necessary, as the patient's own musculature splints well enough. With multiple rib fractures, the patient can develop ventilation problems and should be hospitalized.

\section{Hyperventilation}

Acute and chronic hyperventilation syndromes generally have a significantly decreased arterial $\mathrm{PCO}_{2}$ and respiratory alkalosis. The diagnosis does not require measurement of arterial blood gases, as the clinical signs and symptoms should prompt the clinician to have the patient hyperventilate in a controlled circumstance to produce the chief complaints (provocation test). The rebreathing of higher concentrations of carbon diox- 
ide (by breathing in and out of a bag) relieves acute and/or chronic symptoms. If patients can be shown the cause of their symptoms with a provocation test, most improve. ${ }^{76}$ In the long run, efforts to train patients to breathe more slowly and abdominally may also be successful. ${ }^{77}$ The behavioral and psychiatric aspects of hyperventilation are discussed in Chapter 32 .

\section{Rare Pulmonary Diseases Encountered by the Family Physician}

The family physician uncommonly sees a patient with an abnormal infiltrate on chest $\mathrm{x}$-ray film and eosinophilia on the blood smear. Pulmonary infiltrations associated with blood eosinophilia (PIE syndrome) range from simple pulmonary eosinophila, or Löffler's syndrome, through eosinophilic pneumonia to polyarteritis nodosa. Löffler's syndrome has mild symptoms: cough, chest discomfort, and malaise. The disease lasts less than a month, and the chest $\mathrm{x}$-ray film shows unilateral or bilateral homogeneous infiltrates. Eosinophil counts can be as high as $20 \%$ with leukocytosis. This benign disease regresses without therapy.

Eosinophilic pneumonia lasts upward of 6 months. Fevers and cough are common. The chest x-ray film shows bilateral, usually upper lobe, vague infiltrates that come and go. Chronic eosinophilic pneumonia is usually treated with corticosteroids for 1 to 2 months.

Polyarteritis nodosa is a serious and often fatal disease. Infiltrates seen on chest $\mathrm{x}$-ray films are vague, and they come and go. A biopsy is needed to make the diagnosis of polyarteritis nodosa. Small arteries in the lung and elsewhere are involved with fibrinoid changes and, at times, giant cells with neutrophils and eosinophils. Long-term, high-dose corticosteroid treatment is indicated.

Bronchiolitis obliterans is an uncommon disease in which the smaller bronchioles are obliterated by an inflammatory reaction. Viral infections and toxins such as chlorine can produce such lesions. Patients present with breathlessness and hyperinflation (seen on the chest $\mathrm{x}$-ray film). An open lung biopsy is required for diagnosis. Corticosteroids during the active process may help.

Wegener's granulomatosis is a severe ulcerating disease of the lungs, upper respiratory areas, and kidneys. It presents with infiltrates, cavities, or both on the chest $\mathrm{x}$-ray film. An open lung biopsy is required for diagnosis. Cytoxan, methotrexate, or trimethoprim-sulfamethoxazole ${ }^{78}$ has induced remissions.

Pulmonary alveolar proteinosis is a rare disease with an $\mathrm{x}$ ray appearance resembling that of pulmonary edema, with the lungs full of material that is strongly positive to periodic acidSchiff (PAS) staining. Bronchoalveolar lavage can establish the diagnosis and is also used for treatment.

Atypical mycobacteria can cause a tuberculous-like picture, particularly in those with underlying immune deficiencies. The two most common causative organisms are Mycobacterium kansasii and Mycobacterium avium-intracellulare. The first is usually sensitive to rifampin, the latter to nothing. $M$. avium-intracellulare is usually superimposed on COPD, old tuberculosis, bronchiectasis, or AIDS. The organism must be found on repeated sputum cultures (smears are less reliable) in the face of significant clinical disease. Blood cultures are often positive in AIDS patients. Treatment is with up to six antituberculous agents. In those with AIDS, treatment is controversial but clofazimine (an antileprosy agent) and ansamycin (an experimental rifampin derivative) are sometimes included. Therapy is usually continued for 18 to 24 months. The prognosis for AIDS patients is dismal; for others the disease has a slow and chronic course. For patients with localized disease, resectional surgery combined with chemotherapy may offer a better outcome. ${ }^{79}$

\section{Preoperative Pulmonary Evaluation}

Work-up guidelines for thoracoabdominal surgery include screening spirometry. If it is abnormal, the patient is placed on beta- 2 agonist therapy, with an absolute contraindication for smoking for at least 1 month prior to surgery. If the FEV is $<1$ liter or $<50 \%$ predicted, pulmonary consultation is indicated. The presence of a concurrent upper or lower respiratory tract infection postpones any elective surgery. A preventive approach with bronchodilators and cessation of smoking reduces the postoperative complications of atelectasis, hypoxemia, and pneumonia.

\section{Air Travel for the Patient with Lung Disease}

Commercial airlines usually fly at cabin pressures of 5,000 to 8,000 feet. It is important for the patient with chronic lung disease who is hypoxemic at sea level on room air to know if supplementary oxygen is needed during air travel at these altitudes. At sea level a person has an arterial $\mathrm{PO}_{2}$ level of approximately $95 \mathrm{~mm} \mathrm{Hg}$, and at 5,000 feet it decreases to about $75 \mathrm{~mm} \mathrm{Hg}$. An arterial $\mathrm{PO}_{2}$ of $60 \mathrm{~mm} \mathrm{Hg}$ in a chronic lung patient on room air at sea level decreases to approximately $50 \mathrm{~mm} \mathrm{Hg}$ at 5,000 feet. In general, patients who at sea level on room air have an arterial $\mathrm{PO}_{2}$ of $<60 \mathrm{~mm} \mathrm{Hg}$ are considered candidates for supplemental oxygen at 1 to $3 \mathrm{l} / \mathrm{min}$ during commercial air travel. The same guidelines pertain for patients who are moving to or traveling through areas of high altitude. For specific recommendations in an individual patient, altitudes can be simulated with an oxygen-nitrogen blend so that by using oximetry the percent oxygen-hemoglobin saturation can be determined for any altitude. Most major airlines make special arrangements for passengers who require supplemental oxygen, but is is necessary to notify the airline ahead of time. Many airlines request a statement signed by a physician stating that the patient requires a specific flow rate of oxygen and that the patient is able to fly without undue risk. ${ }^{80}$

\section{Core Procedures}

\section{Chest Roentgenogram Interpretation}

The posteroanterior (PA) chest $\mathrm{x}$-ray film is the most frequently ordered radiographic examination. Indications for a chest film depend on the clinical suspicion of active disease based on a positive history and physical findings. Routine chest films on hospital admission, for screening smokers for lung cancer or for follow-up of treated tuberculosis, are not 
indicated. The prevalence of clinically important findings on chest films in asymptomatic patients is less than $0.5 \%{ }^{81}$

There is no absolute or correct way to read chest films, but the following is presented as a useful, step-by-step approach ${ }^{81}$ Start by checking the name and date on the film. Then stand back and get a "gestalt" of the film. If any abnormality stands out, look away from it so as not to jump to conclusions. Next, focus closer and follow these steps, which are illustrated in Figure 17.1.

1. Are costophrenic angles sharp? Is there any small pleural effusion?

2. Look along both diaphragms. Are they smooth and the same height? (Usually the right is slightly higher.) Look for a stomach gas bubble.

3. Look at the chest wall. Is the pleura thickened? Examine each rib individually to look for old or new fractures or rib notching. Look for cervical ribs. Look for the breast shadows. Removal of breast tissue may make one lung appear darker or more translucent. Look at the axilla and supraclavicular area for any soft tissue abnormalities, asymmetry, or calcifications.

4. Look at the superior mediastinum. Is the trachea in the middle? Follow it from the neck down to the carina. Is the trachea smooth and not narrowed? Look at the hilar areas. Are they enlarged, or are there calcifications? Usually the left hilar shadow is slightly higher than the right. Is there an azygous lobe on the right? Is the aorta widened? Is it tortuous? Are there calcifications in the intima?

5. Look at the heart outline. Trace the outline, starting at the right cardiophrenic angle: right atrium, superior vena cava, aorta, left atrium, and left ventricle. A rough guide for heart size is that the greatest transverse diameter of the heart should be less than $50 \%$ of the intrathoracic diameter (as measured from one costophrenic angle to the other).
6. The lung fields are viewed. In the inner zone (A, Fig. $17.1)$, one predominantly sees pulmonary vessels that are $<2$ $\mathrm{cm}$ in diameter. In the middle (B) zone, vessels seldom exceed $5 \mathrm{~mm}$ in diameter. In the outer one-third (C) zone, only the fine reticular markings should be visible all the way to the chest wall. In the standing position, blood flows preferentially to the lower lobes, and vessels there stand out and are larger than in the upper lobes. Upper lobe vessels that appear $>5$ $\mathrm{mm}$ in diameter may indicate venous hypertension. Look for the sulci and fissures. Look at each lobe for consolidations or nodules. Look for Kerley B lines or short peripheral, horizontal lines that may indicate interstitial edema. Small calcifications or nodules may be difficult to differentiate from vessels on end. Look at the density (calcifications are denser) and the contours (vesseis are smooth); and look carefully for branching vessel shadows leading to the nodule.

7. View the remaining bones, including thoracic and cervical spine, scapula, clavicles, and what is visible of the shoulder joints.

8. If any old films are available, place them side by side with the new ones and compare abnormalities. Previous chest films have probably saved more people from unnecessary procedures than any other radiographic test.

\section{Tuberculin Skin Test}

Theory has it that if the tuberculin skin test is positive, there are live mycobacteria in the body: It implies tuberculosis exposure sometime in the past. Indications for the skin test include contact investigation and screening for communicable disease in high risk populations such as health care workers, individuals born and/or raised in Third World countries, jail inmates, and the elderly. Previous Bacillus Calmette Guérin (BCG) vaccination is not a contradiction. Routine tuberculosis skin testing is not indicated when examining low risk

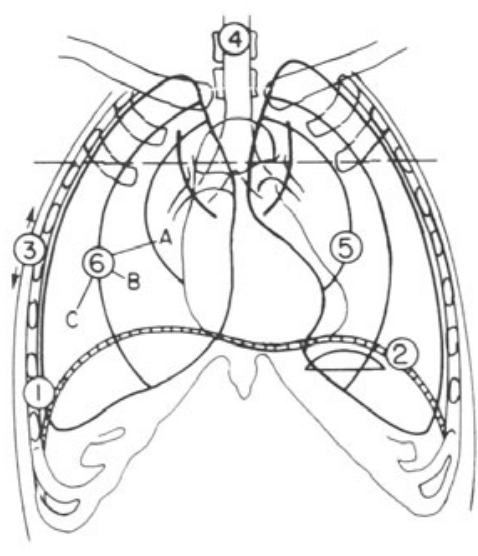

Fig. 17.1 Steps to follow when reading the posteroanterior (PA) chest X-ray film 1, Costophren, sinuses; 2, diaphragm level (and gas bubble); 3, chest wall (axillas, pect. $\mathrm{mm}$. breast); 4, neck and trachea; 5, mediastinum and heart and major vessels and azygus; 6 , three zones of lung. Anatomic relations of arteries, veins, bronchi, bones, and soft tissue
Apical artery (right) $\quad$ Trachea
Apical vein (right)

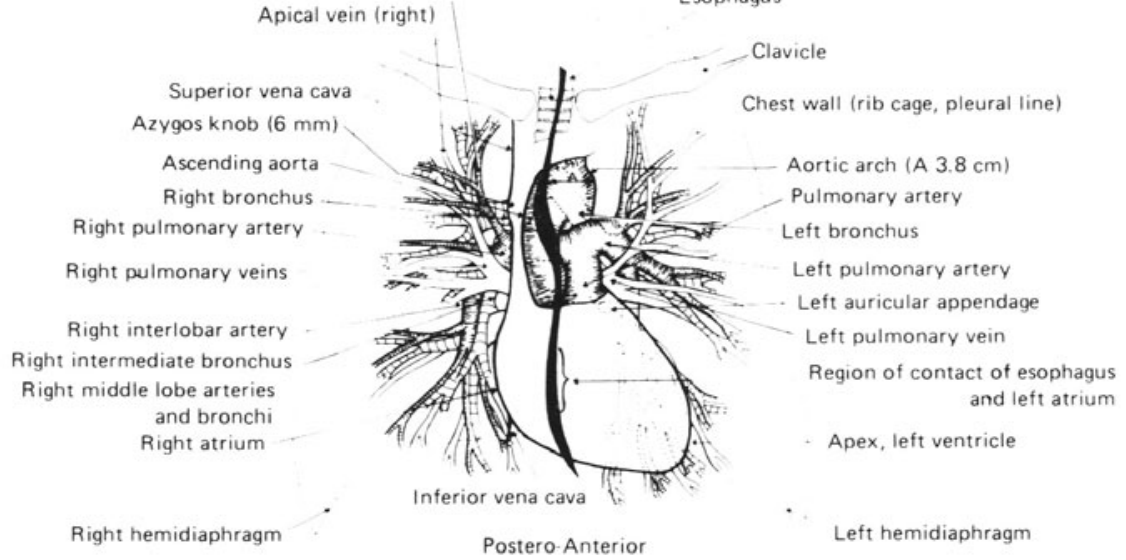

structures on the PA chest X-ray film. Reprinted with permission from Meschan I, Ott DJ. Introduction to Diagnostic Imaging. Philadelphia: W.B. Saunders, 1984, and Meschan I. Synopsis of Radiologic Anatomy with Computed Tomography. Philadelphia: W.B. Saunders, 1980. 
groups, as in well-baby care of most children born in the United States. ${ }^{82}$ The incidence of a positive skin test varies from $0.1 \%$ or less in those under age 20 , to 10 to $20 \%$ in the elderly, to more than $50 \%$ in some refugee groups.

The intradermal (Mantoux) skin test is the "gold standard." It is administered using $0.1 \mathrm{cc}$ of intermediate PPD-T (Tweenstabilized purification protein derivative) ( $5 \mathrm{TU}$ ) injected intradermally. PPD-T is an extract of a culture of mycobacteria. Interestingly, when its precursor (old tuberculin, or OT) was discovered by Koch in 1890 , it was thought to cure tuberculosis.

The technique is to hold the syringe parallel to the skin surface with the bevel of the 27-gauge needle facing upward, enter and then inject slowly just below the skin surface. A small bleb forms. If a small amount goes subcutaneously (too deeply), it is all right. The bleb is absorbed in an hour or so.

The skin test is read in 48 to 72 hours. The amount of erythema is not crucial, but a lot usually indicates a positive test. What is recorded is induration. One needs practice to read the test accurately, and various techniques have been suggested: running one's finger gently across the area to feel for increased thickness, viewing the arm laterally (one can usually see an elevation), rolling the skin lightly between fingers to feel the induration, or slowly running a ballpoint pen across the area to feel where it becomes slightly more difficult to push. Lastly, a plastic calipers (originally developed by the Oregon Thoracic Society) give reproducible readings. A positive test is an induration of $10 \mathrm{~mm}$ or more in diameter. Although this criterion is arbitrary, it is a good compromise for sensitivity and specificity. The larger the induration, the more likely it is caused by tuberculosis and does not represent cross-reactivity from atypical strains.

Three intradermal multiple-puncture methods are also available: (1) the tine test, which uses four prongs coated with OT or PPD-T; (2) the Aplitest, which uses four prongs covered with PPD-T; and (3) the Monovac, which uses nine prongs and a small vial of OT that is applied to the prongs just before using. Supposedly, the latter gets around any problems of not having the prongs adequately coated. These tests are also read at 48 to 72 hours; but the reading is often difficult, and therein lies a problem. Although a positive test is usually defined as $>$ $2 \mathrm{~mm}$ induration, any erythema calls for retesting with intradermal PPD-T. Because of the small size of the test area and varied reactions, the authors believe it is unreliable to have family members read the results and call or send them in. Now that frequent skin testing of children is not needed in most parts of the United States, the use of these other skin tests should decrease and they will be replaced by the Mantoux test, which must be administered and read by an experienced person. Atypical, first, and second-strength PPD preparations are no longer available.

Many years after infection, skin test reactivity may fade (as it does with BCG vaccination). The initial skin test may be borderline or negative, but one repeated weeks to months later is positive. It is called the booster phenomenon and does not represent recent conversion. In the elderly and in hospital employees, it is often recommended that one skin test be given perhaps without even reading it; instead, read carefully a second test given 2 to 4 weeks later. This procedure reduces the number of converters found in subsequent annual screenings.

\section{Pulmonary Function Testing}

Office spirometry is needed for the diagnosis and follow-up of patients with obstructive airways diseases. As $10 \%$ of the population has some form of obstructive airway diseases, every family physician's office should have a simple spirometry apparatus. A bellows-type spirometer that gives a paper readout is preferred. The shape of the spirometry curve is helpful, and it serves as a hard copy for the chart. Spirometry gives reproducible data regarding the forced vital capacity (FVC) and the $\mathrm{FEV}_{1}$. Of these values, the $\mathrm{FEV}_{1}$ value is the most important (equally important is the percent of the $\mathrm{FEV}_{1} / \mathrm{FVC}$ ). The values of $\mathrm{FEV}_{1}$ and $\mathrm{FVC}$ are easily measured on the paper readout by reading the value on the vertical axis where the curve is at 1 second and the maximum height of the curve, usually at 3 to 5 seconds (Fig. 17.2). These values are then compared to a nomogram or chart that takes into account the patient's gender, age, and height to give the percent of predicted age value for that individual. Normal values are considered within $20 \%$ of predicted ones.

To obtain accurate measurements, it is important to use a nose clip and to encourage maximal effort. If abnormal, one should also retest 15 minutes after the patient used an inhaled bronchodilator for reversibility of the defect. When spirometry does not clearly differentiate between obstruction and restrictive lung disease, lung volume studies are needed.

\section{Thoracentesis and Chest Intubation}

Thoracentesis is a procedure the family physician should be able to perform. It is used to (1) relieve symptoms by draining off pleural fluid or (2) make a diagnosis by studying pleural fluid. The patient should be sitting upright with the head

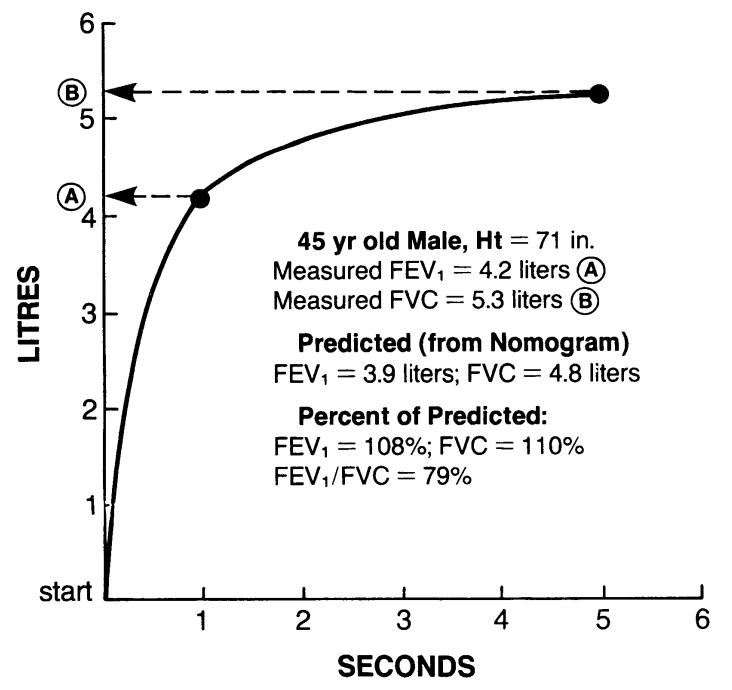

Fig. 17.2. Graph of a spirometry printout from a normal man. Point $\mathrm{A}$ is the reading of forced expiratory volume in one second $\left(\mathrm{FEV}_{1}\right)$. Point $\mathrm{B}$ is the forced vital capacity (FVC). 
supported on a pillow on a portable stand. A disposable kit is generally used that contains a catheter to be placed in the pleural space. After reviewing the chest X-ray films, one carefully palpates and percusses to find the top of the ribs in the posterior axillary line at an interspace judged to be beneath the meniscus of the pleural effusion and above the diaphragm. If needed, ultrasound guidance is helpful. After local anesthesia the pleural space is entered with a needle puncture; the catheter is left in the pleural space, and the needle is removed. The authors prefer the catheter to be larger, sliding over the needle, in contrast to one that is smaller and within the needle, as catheters within needles may be cut off. The removal of moderate amounts of pleural fluid at a time is recommended, as the abrupt removal by vacuum-type bottles causes pain and may produce a pneumothorax because the lung cannot expand fast enough to fill the space being created. Rarely, patients develop unilateral pulmonary edema when the lung cannot expand fast enough to fill the pleural space with the rapid removal of a large pleural effusion. If air appears in the pleural space, an attempt is made to aspirate it before removing the catheter. A chest film is obtained after each thoracentesis to evaluate the small pneumothorax that can result. The risk of pneumothorax with thoracentesis is about 5 to $10 \%$ although most of these patients do not require a chest tube.

The pleural fluid is tested for total cell count and differential, glucose, protein concentration and lactic dehydrogenase; Gram's staining and aerobic and anaerobic cultures with sensitivity tests are performed. Other tests to be considered are $\mathrm{pH}$; cytology; tuberculous, or fungal cultures; antinuclear antibody, and rheumatoid factor.

If the pleural fluid is a transudate with a protein content of $<3 \mathrm{~g} / \mathrm{dl}$, the differential diagnosis is narrowed to congestive heart failure, liver failure, kidney failure, or hypothyroidism. If the fluid is an exudate with a protein content of $>3 \mathrm{~g} / \mathrm{dl}$, the differential diagnosis is greatly expanded into a long list of inflammatory and neoplastic causes.

If a chest tube is needed to remove air or fluid, it is inserted into the fourth or fifth intercostal space in the anterior axillary line (just lateral to the pectoral fold) by an initial skin incision followed by blunt dissection to the pleural space. Forceps entry is then made in a blunt manner into the pleural space, followed by finger exploration. One needs to be sure that there are no adhesions, and that there is a pleural space in which to insert the chest tube. Tubes for "air" are directed toward the apex, and tubes for "fluid" are directed toward the posteroinferior mid-chest. Chest tubes go their own way once they leave the physician's guiding fingers. The tube is connected to a drainage system, which may vary from a single water seal bottle to a one-piece plastic drainage system that can be connected to wall suction, or it can be left to room air. Chest tubes are left in place until they have done the function asked of them-removal of air or fluid-and then they are removed. The chest tube suction is turned off and the tube is connected to a water seal before removal; and then if there is no evidence of leaking air or reaccumulation of fluid, the tube is removed with a follow-up chest film.

In cases of pneumothorax, a smaller tube may be inserted anteriorly over the upper anterior chest ribs by percutaneous technique, connecting the tube to a one-way Heimlich valve.
Such patients can be sent home and followed if they are reliable.

\section{Gram's Stain of Infected Material (Sputum)}

The first, most important step of the Gram's stain, which owes its name and discovery to Christian Gram (1853-1938), is collection of an adequate sample. The physician at the side of a patient with pneumonia should oversee the expectoration and collection of the sample. The sample is then sent promptly to the laboratory for a Gram's stain.

Once a sample has been properly obtained and stained, the microscopic examination concentrates on whether it is an adequate specimen. If the sputum has $>25$ squamous epithelial cells per low power $(100 \times)$ magnification field, the sample is discarded as being from the upper respiratory tract. If the sample has $<25$ squamous epithelial cells per field (shed from buccal mucosa), and if it shows $>10$ polymorphonuclear leukocytes (PMNs) per sample field, it is viewed more carefully. The original sample can be cultured if needed. Sputum implies a lower respiratory tract sample. If the sputum sample is considered adequate in terms of the presence of polymorphonuclear leukocytes, it is inspected for stained bacteria near or in the PMNs. If such bacteria are scanty or rare, the conclusion is that a nonbacterial process, i.e., viral or mycoplasmal, is the cause. If bacteria are plentiful, the Gram-stained types are noted. A predominance of one type $(>50 \%)$ suggests an infection of that type, i.e., gram-positive diplococci (pneumococcus); gram-positive grape-like cocci, often intracellular (Staphylococcus); gram-negative small pleomorphic bacilli (H. influenzae); or thick gram-negative bacilli (Klebsiella). Sputum cultures then follow the Gram's stain if appropriate.

\section{References}

1. Marsland DW, Wood M, Mayo F. Content of family practice. 1. Rank order of diagnosis by frequency. J Fam Pract 1976;3:37-47.

2. National Center for Health Statistics. 1981 office visits for general and family practitioners. Washington, DC: NCHSR, US Department of HHS.

3. Shear CL, Wall GM. Diagnosis cluster frequency in a communitybased family practice residency program. West $\mathbf{J}$ Med 1985; 142:854-7.

4. Rodnick JE. Unpublished data. 1985.

5. Stott NCH, West RR. Randomized controlled trial of antibiotics in patients with cough and purulent sputum. Br Med J 1976;2: $556-9$.

6. Williamson HA. A randomized, controlled trial of doxycycline in the treatment of acute bronchitis. J Fam Pract 1984;19:481-6.

7. Franks P, Gleiner JA. The treatment of acute bronchitis with trimethoprim and sulfamethoxazole. J Fam Pract 1984;19:185-90.

8. Brickfield FX, Carter WH, Johnson RE. Erythromycin in the treatment of acute bronchitis in a community practice. J Fam Pract 1986;23:119-22.

9. Gleckman RA. Acute and chronic bronchitis and acute infectious exacerbations of chronic disease. Fam Pract Recert 1985;7:81-92.

10. Pennington JE. Treating respiratory infections in the era of cost control. Am Fam Physician 1986;33:153-61.

11. Traver GA, Cline MG, Burrows B. Predictors of mortality in chronic obstructive pulmonary disease. Am Rev Respir Dis 1979;119:895-902.

12. Nenery B, Moavero NE, Brasseur L, Stanescu DC. Changes in lung function after smoking cessation: an assessment from a cross-sectional survey. Am Rev Respir Dis 1982;125:122-124. 
13. Cummings SR. Kicking the habit: benefits and methods of quitting cigarette smoking. West J Med 1982;137:442-7.

14. "Nicotine chewing gum" (editorial). Lancet 1985;1:320-1.

15. Bouschey HA, Holtzman MJ, Sheller JR, Nadel JA. State of the art-bronchial hyperreactivity. Am Rev Respir Dis 1980;121: 389-413.

16. Sheppard D. Pharmacotherapy of asthma. West J Med 1985;142:700-2.

17. Eaton ML, Green BA, Church TR, McGowan T, Niewoehner DE. Efficacy of theophylline in "irreversible" airflow obstruction. Ann Intern Med 1980;92:758-61.

18. Albert RK, Martin TR, Lewis SW. Controlled clinical trial of methylprednisolone in patients with chronic bronchitis and acute respiratory insufficiency. Ann Intern Med 1980;92:753-8.

19. Nicotra MG, Rivera M, Awe RJ. Antibiotic therapy of acute exacerbations of chronic bronchitis-a controlled study using tetracycline. Ann Intern Med 1982;97:18-21.

20. Petrie GR, Palmer KNV. Comparison of aerosol ipratropium bromide and salbutamol in chronic bronchitis and asthma. Br Med J $1975 ; 1: 430-2$

21. Nocturnal Oxygen Therapy Trial Group. Continuous or nocturnal oxygen therapy in hypoxemic chronic obstructive lung disease. Ann Intern Med 1980;93:391-8.

22. Lertzman MM, Cherniack RM. State of the art-rehabilitation of patients with chronic obstructive pulmonary disease. Am Rev Respir Dis 1976;114:1145-65.

23. Pardy RL, Rivington RN, Despas PJ, Macklem PT. Inspiratory muscle training compared with physiotherapy in patients with chronic air flow limitation. Am Rev Respir Dis 1981;123:421-5.

24. La Force FM. Community-acquired lower respiratory tract infections. Am J Med 1985;78(suppl. 6B):52-7.

25. Fick RB, Reynolds HY. Changing spectrum of pneumonia-news media creation or clinical reality? Am J Med 1983;74:1-8

26. Davidson M, Tempest B, Palmer DL. Bacteriologic diagnosis of acute pneumonia-comparison of sputum, transtracheal aspirates and lung aspirates. JAMA 1976;235:158-63.

27. Health and Public Policy Committee, American College of Physicians. Pneumococcal Vaccine. Ann Intern Med 1986;104:118-20.

28. Tuazon CU. Gram-positive pneumonias. Med Clin North Am 1980;64:343-61.

29. Reyes MP. The aerobic gram-negative bacillary pneumonias Med Clin North Am 1980;64:363-83.

30. Murray HW, Tuazon C. Atypical pneumonias. Med Clin North Am 1980;64:507-27.

31. Cordes LG, Fraser DW. Legionellosis. Med Clin North Am 1980;64:395-416.

32. Grayston JT, Kuo C, Wang S, Altman J, A new Chlamydia psittaci strain, TWAR, isolated in acute respiratory tract infections. $\mathrm{N}$ Engl J Med 1986;315:161-8.

33. Beem MO, Saxon E, Tipple MA. Treatment of chlamydial pneumonia of infancy. Pediatrics 1979;63:198-203.

34. Irwin RS, Corrao WM, Pratter MR. Chronic persistent cough in the adult: the spectrum and frequency of causes and successful outcome of specific therapy. Am Rev Respir Dis 1981;123:413-17.

35. Stulbarg M. Evaluating and treating intractable cough. West $J$ Med 1985;143:223-8.

36. Irwin RS, Rosen MJ, Braman SS. Cough, a comprehensive review. Arch Intern Med 1977;137:1186-91.

37. Jett JR, Cortese DA, Fontana RS. Lung cancer: current concepts CA 1983;33:74-86.

38. Heitzman ER. The role of computed tomography in the diagnosis and management of lung cancer-an overview. Chest 1986;89:237S-41S.

39. Gamsu G. Magnetic resonance imaging in lung cancer. Chest 1986;89:242S-4S.

40. Carr DT, Mountain CF. Staging of lung cancer. Semin Respir Med 1982;3:154-63.

41. Dalen JE. Alpert JS. Natural history of pulmonary embolism. Prog Cardiovasc Dis 1975;17:259-70.

42. Fulkerson WJ, Coleman RE, Ravin CE, Saltzman HA. Diagnosis of pulmonary embolism. Arch Intern Med 1986;146:961-7.

43. Hull RD, Hirsh J, Carter CJ, et al. Diagnostic efficacy of impedance plethysmography for clinically suspected deep-ven- ous thrombosis-a randomized trial. Ann Intern Med 1985;102: 21-8.

44. The Urokinase Pulmonary Embolism Trial-A National Cooperative Study. Circulation 1973;47(suppl. II):1-108.

45. Hull R. Delmore T, Carter O, et al. Adjusted subcutaneous heparin versus warfarin sodium in the long-term treatment of venous thrombosis. N Engl J Med 1982;306:189-94.

46. Petitti DB, Stom Bl, Melmon KL. Duration of warfarin anticoagulant therapy and the probabilities of recurrent thromboembolism and hemorrhage. Am J Med 1986;81:255-9.

47. An International Multicentre Trial. Prevention of fatal postoperative pulmonary embolism by low doses of heparin. Lancet $1975 ; 2: 45-51$

48. Snider DE, Cohn DL, Davison PT, Hershfield ES, Smith MH, Sutton FD. Standard therapy for tuberculosis 1985. Chest $1985 ; 87: 117$ S-24S.

49. Farer LS. Tuberculosis, what the clinician should know. New York: American Lung Association, 1982.

50. Luce JM. Interstitial lung disease. Hosp Pract 1983;July:173-89.

51. Fulmer JD. The interstitial lung diseases. Chest 1982;82:172-8.

52. Mazzocco MC, Owens GR, Kirilloff LM, Roger RM. Chest percussion and postural drainage in patients with bronchiectasis. Chest 1985;88:360-63.

53. Ellis DA, Thornley PE, Wightinan AJ, et al. Present outlook in bronchiectasis: clinical and social study and review of factors influencing prognosis. Thorax 1981;36:659-64.

54. Wohl ME, Chernick V. Bronchiolitis: state of the art. Am Rev Respir Dis 1978;118:759-81.

55. Wright PF. Bronchiolitis. Pediatr Rev 1986;7:219-22.

56. Webb MSC, Henry RL, Milner AD, Stokes GM, Swarbick AS Continuing respiratory problems three and a half years after acute viral bronchiolitis. Arch Dis Child 1985;60:1064-7.

57. Catanzaro A, Einstein H, Levine B, Ross B, Schillaci R, Fierer J. Ketoconazole for treatment of disseminated coccidioidomycosis. Ann Intern Med 1982;96:436-40.

58. Stamm AM, Dismukes WE. Current therapy of pulmonary and disseminated fungal diseases. Chest 1983;83:911-7.

59. Goodwin RA, Des Pres RM. Histoplasmosis: state of the art. Am Rev Respir Dis 1978;117:929-56.

60. Penn RL, Lambert RS, George RB. Invasive fungal infections: the use of serologic tests in diagnosis and management. Arch Intern Med 1983;143:1215-20.

61. Sarosi GA, Davies SF. Blastomycosis: state of the art. Am Rev Respir Dis 1979;120:911-38.

62. Sarosi GA. Management of fungal disease. Am Rev Respir Dis $1983 ; 127: 250-3$

63. Wang JLF, Patterson R, Rosenberg M, Roberts M, Cooper BJ Serum IgE antibody activity against Aspergillus fumigatus as a diagnostic aid in allergic bronchopulmonary aspergillosis. Am Rev Respir Dis 1979;117:917-27.

64. Binder RE, Faling LJ, Pugatch RD. Chronic necrotizing pulmonary aspergillosis: a discrete clinical entity. Medicine 1982;61:109-24.

65. Kerkering TM, Duma RJ, Shadomy S. The evolution of pulmonary cryptococcosis. Ann Intern Med 1981;94:611-16.

66. Petersen EA, Nash ML, Mammana RB, Espeland JG. Minocycline Treatment of pulmonary nocardiosis. JAMA 1983;250: 930-2.

67. Bascom R, Johns CJ. The natural history and management of sarcoidosis. Adv Intern Med 1986;31:213-41.

68. Daniele RP, Dauber JH, Rossman MD. Immunologic abnormalities in sarcoidosis. Ann Intern Med 1980;92:406-16.

69. Puar HS, Young RC, Armstrong EM. Bronchial and transbronchial lung biopsy without fluoroscopy in sarcoidosis. Chest 1985;87:303-6.

70. Rom WN, Lockey JE. Diffuse malignant mesothelioma: a review. West J Med 1982;137:548-54.

71. DiGregorio GJ, Kotyuk BL. Toxicology of asbestos. Am Fam Physician 1985;32:201-4.

72. Kales A, Soldatos CR, Kales JD. Taking a sleep history. Am Fam Physician 1980;22:101-9.

73. Tobin ML, Cohn MA, Sackner MA. Breathing abnormalities during sleep. Arch Intern Med 1983;143:1221-8. 
74. Fairbanks DNF. Snoring: not funny-not hopeless. Am Fam Physician 1986;33:205-11.

75. Jenkinson SG. Pneumothorax. Clin Chest Med 1985;6:153-61.

76. Magarian GJ. Hyperventilation syndromes: infrequently recognized common expressions of anxiety and stress. Medicine $1982 ; 61: 219-36$.

77. Grossman P, de Swart JC, Defares PB. A controlled study of a breathing therapy for treatment of hyperventilation syndrome. $\mathrm{J}$ Psychosom Res 1985;29:49-58.

78. West BC, Todd JR, King JW. Wegeners granulomatosis and trimethoprim-sulfamethoxazole. Ann Intern Med 1987;106:840-2.
79. Iseman MD, Corpe RF, O'Brien RJ, Rosenzweig DY, Wolinsky E. Disease due to Mycobacterium avium-intracellulare. Chest 1985;87:1395-1495.

80. Voss MW. Air travel for the chronically ill and the elderly. Am Fam Physician 1983;27:235-243.

81. Tape TG, Muslin AI. The utility of routine chest radiographs. Ann Intern Med 1986;104:663-70.

82. Kearns TJ, Cole CH, Farer LS, et al. Public health issues in control of tuberculosis. Chest $1985 ; 87: 135 \mathrm{~S}-8 \mathrm{~S}$. 